\title{
FROM RINGS TO BULGES: EVIDENCE FOR RAPID SECULAR GALAXY EVOLUTION AT $z \sim 2$ FROM INTEGRAL FIELD SPECTROSCOPY IN THE SINS SURVEY ${ }^{1}$
}

\author{
R. Genzel, ${ }^{2,3}$ A. Burkert, ${ }^{4}$ N. Bouché, ${ }^{2}$ G. Cresci, ${ }^{2}$ N. M. Förster Schreiber, ${ }^{2}$ A. Shapley, ${ }^{5}$ K. Shapiro, ${ }^{6}$ \\ L. J. Tacconi, ${ }^{2}$ P. Buschkamp, ${ }^{2}$ A. Cimatti,${ }^{7}$ E. Daddi,${ }^{8}$ R. Davies, ${ }^{2}$ F. Eisenhauer, ${ }^{2}$ D. K. Erb, ${ }^{9}$ \\ S. Genel, ${ }^{2}$ O. Gerhard, ${ }^{2}$ E. Hicks, ${ }^{2}$ D. Lutz, ${ }^{2}$ T. NaAb, ${ }^{4}$ T. Ott, ${ }^{2}$ S. Rabien, ${ }^{2}$ \\ A. Renzini, ${ }^{10}$ C. C. Steidel, ${ }^{11}$ A. Sternberg, ${ }^{12}$ and S. J. Lilly ${ }^{13}$ \\ Received 2008 April 13; accepted 2008 July 7
}

\begin{abstract}
We present $\mathrm{H} \alpha$ integral field spectroscopy of well-resolved, UV/optically selected $z \sim 2$ star-forming galaxies as part of the SINS survey with SINFONI on the ESO VLT. Our laser guide star adaptive optics and good seeing data show the presence of turbulent rotating star-forming outer rings/disks, plus central bulge/inner disk components, whose mass fractions relative to the total dynamical mass appear to scale with the $[\mathrm{N}$ II $] / \mathrm{H} \alpha$ flux ratio and the star formation age. We propose that the buildup of the central disks and bulges of massive galaxies at $z \sim 2$ can be driven by the early secular evolution of gas-rich proto-disks. High-redshift disks exhibit large random motions. This turbulence may in part be stirred up by the release of gravitational energy in the rapid "cold" accretion flows along the filaments of the cosmic web. As a result, dynamical friction and viscous processes proceed on a timescale of $<1 \mathrm{Gyr}$, at least an order of magnitude faster than in $z \sim 0$ disk galaxies. Early secular evolution thus drives gas and stars into the central regions and can build up exponential disks and massive bulges, even without major mergers. Secular evolution along with increased efficiency of star formation at high surface densities may also help to account for the short timescales of the stellar buildup observed in massive galaxies at $z \sim 2$.
\end{abstract}

Subject headings: cosmology: observations — galaxies: evolution — galaxies: high-redshift — infrared: galaxies Online material: color figures

\section{INTRODUCTION}

Deep surveys have become efficient in detecting $z \sim 1.5-3.5$ star-forming galaxy populations near the peak of cosmic star formation and QSO activity (e.g., Steidel et al. 1996, 2004; Franx et al. 2003; Daddi et al. 2004a). Large samples are now available, based on their rest-frame UV magnitude/color properties (the socalled "BX/BM" criterion: Adelberger et al. 2004; Steidel et al. 2004), or on rest-frame optical magnitude/color properties ("starforming" or "s"'-BzKs; Daddi et al. 2004a, 2004b; DRGs: Franx et al. 2003). These selection criteria sample fairly luminous $\left(L \sim 10^{11}-10^{12.5} L_{\odot}\right)$ galaxies with star formation rates of

\footnotetext{
1 Based on observations at the Very Large Telescope (VLT) of the European Southern Observatory (ESO), Paranal, Chile.

2 Max-Planck-Institut für extraterrestrische Physik (MPE), Giessenbachstr.1, D-85748 Garching, Germany; genzel@mpe.mpg.de.

3 Department of Physics, Le Conte Hall, University of California, Berkeley, CA 94720.

4 Universitäts-Sternwarte Ludwig-Maximilians Universität (USM), Scheinerstr. 1, München, D-81679, Germany.

5 Department of Physics and Astronomy, 430 Portola Plaza, University of California, Los Angeles, CA 90095-1547.

6 Department of Astronomy, Campbell Hall, University of California, Berkeley, CA 94720 .

7 Istituto Nazionale di Astrofisica-Osservatorio Astronomico di Bologna, Via Gobetti 101, I-40129 Bologna, Italy.

8 Service d'Astrophysique, Dapnia CEA, Saclay, France.

9 Harvard-Smithsonian Center for Astrophysics, 60 Garden Street, Cambridge, MA 02138.

10 Osservatorio Astronomico di Padova, Vicolo dell'Osservatorio 5, Padova, I-35122, Italy.

11 California Institute of Technology, MS 105-24, Pasadena, CA 91125.

12 School of Physics and Astronomy, Tel Aviv University, Tel Aviv 69978, Israel.

${ }^{13}$ Institute of Astronomy, Department of Physics, Eidgenössische Technische Hochschule, ETH Zürich, CH-8093, Switzerland.
}

10-300 $M_{\odot} \mathrm{yr}^{-1}$, with a range of ages $10 \mathrm{Myr}-3 \mathrm{Gyr}$ and stellar masses $M_{*} \sim 10^{9}-10^{11.5} M_{\odot}$ (Shapley et al. 2005; Förster Schreiber et al. 2004; Erb et al. 2006b, 2006c; Daddi et al. 2004a, 2004b). These galaxies contribute a large fraction of the cosmic star formation activity and stellar mass density at $z \sim 2$ (Reddy et al. 2005; Rudnick et al. 2006; Grazian et al. 2007).

Still to be determined is how these high- $z$ galaxies formed their stars, when and how the first disks and bulges formed, and how their subsequent evolutions relate to each other. Was the star formation history, and especially the formation of bulges, driven by short, intense bursts following major mergers (mass ratios $<3: 1)$ ? Or was this evolution dominated by a rapid but more continuous "cold" accretion of gas from the dark matter halos, including large mass ratio ( $\geqslant 10: 1)$ gas-rich minor mergers, as proposed by Semelin \& Combes (2002), Birnboim \& Dekel (2003), and Keres et al. (2005)? Motivated by the cold dark matter paradigm of structure formation, the (major) merger interpretation has been favored by many for some time, especially for bulge formation. Recent observations and simulations have lent support to the "rapid accretion" or "cold flow" scenario, at least for the formation of high- $z$ disks. Optically/UV-selected star-forming galaxies at $z \sim 0.5-2.5$ exhibit a fairly tight relationship between stellar mass and star formation rate, arguing against a significant contribution from and time spent in short luminous bursts, rather favoring a high duty cycle of steady star formation, at least for these populations (Noeske et al. 2007; Elbaz et al. 2007; Daddi et al. 2007). Imaging studies have revealed the presence of a number high-z disk galaxies (e.g., Labbé et al. 2003; Stockton et al. 2008). The first spatially resolved studies of the ionized gas kinematics in optically/UV-selected, $z \sim 2$ star-forming galaxies have yielded a surprisingly high abundance of large rotating disks among the more massive $\left(M_{\mathrm{dyn}} \sim 10^{11} M_{\odot}\right)$ and rest-frame optical bright $\left(K_{S} \leqslant 20\right)$ systems. 
TABLE 1

OBserving Log

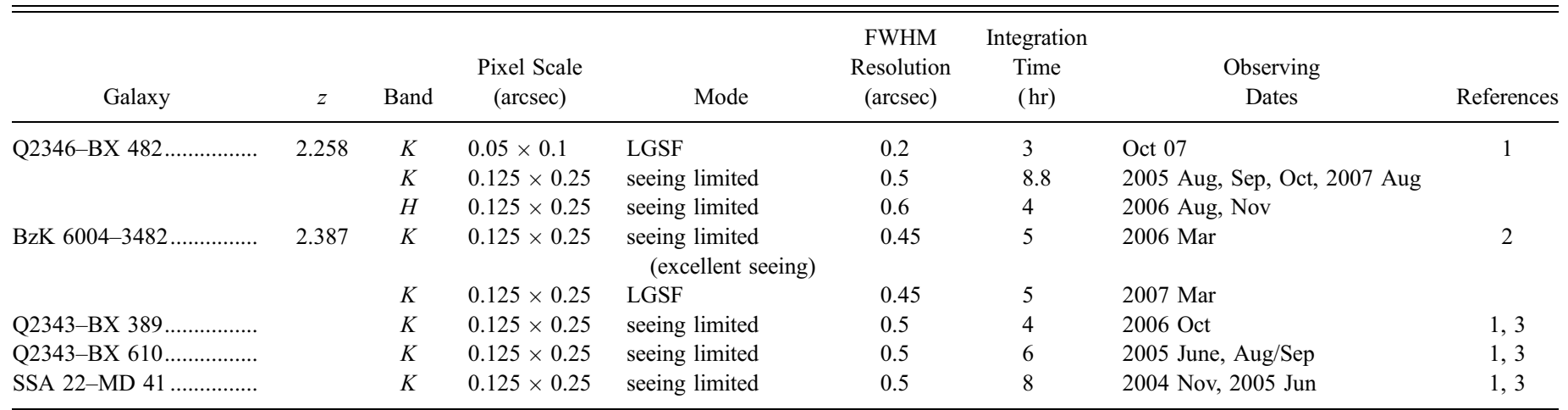

References.-(1) Erb et al. 2006b, 2006c; (2) Kong et al. 2006; (3) Förster Schreiber et al. 2006.

In this subsample only a smaller fraction $(\sim 1 / 3)$ are obvious major mergers (Shapiro et al. 2008; Förster Schreiber et al. 2006; Genzel et al. 2006, Wright et al. 2007; Law et al. 2007; G. Cresci et al. 2008, in preparation). In contrast to these UV/ optically selected galaxies, the very luminous submillimeter galaxies (SMGs) at $z \sim 1-3$ appear to mostly be major mergers in various stages of evolution (Tacconi et al. 2006, 2008; Swinbank et al. 2006). The most recent generation of cosmological galaxy evolution simulations, based on dark matter merger tree simulations, find that smooth accretion of gas and/or minor mergers dominate high- $z$ star formation, while the overall mass assembly of galaxies, and especially the most massive ones, is dominated by mergers (Bower et al. 2006; Kitzbichler \& White 2007; Naab et al. 2007; Guo \& White 2008; Davé 2008; Genel et al. 2008). This conclusion seems to hold for simulations of the baryonic physics based on semianalytic recipes, as well as for hydrodynamic simulations. Taken together, these recent observational and theoretical results are beginning to form a well-formulated framework for the formation of disks, which now needs to be tested by detailed in situ observations. Such measurements also need to explore the evolution of the first disks, as well as the formation bulges.

In this paper we present and analyze high-quality SINFONI/ VLT integral field (IFU) spectroscopy (Eisenhauer et al. 2003; Bonnet et al. 2004) of eight representative UV/optically selected (BX/s-BzK) $z \sim 2$ star forming galaxies. The data for two of these galaxies are discussed here for the first time. For these galaxies we employed laser guide star (LGSF) adaptive optics (AO) to improve the angular resolution. The data for the other six galaxies are discussed in other publications of our team (Förster Schreiber et al. 2006, 2008; Genzel et al. 2006; G. Cresci et al. 2008 , in preparation); here we present a new, detailed analysis. We show that these data deliver interesting new constraints on the issues of disk evolution, bulge formation, and star formation processes. We adopt a $\Lambda$ CDM cosmology with $\Omega_{m}=0.27, \Omega_{b}=$ 0.046 , and $H_{0}=70 \mathrm{~km} \mathrm{~s}^{-1} \mathrm{Mpc}^{-1}$, and a Chabrier (2003) initial stellar mass function.

\section{OBSERVATIONS AND ANALYSIS}

\subsection{Data}

As part of the SINS survey of high- $z$ galaxy kinematics, we observed the $\mathrm{H} \alpha$ and [O III] lines and the rest-frame $R$-band continuum in the UV-selected BX galaxy Q2346-BX $482(z=2.26$; Erb et al. 2006b, 2006c; Förster Schreiber et al. 2006), as well as the $\mathrm{H} \alpha$ line and rest-frame $R$-band continuum in the optically selected s-BzK galaxy BzK 6004-3482 (henceforth BzK
$6004, z=2.39$; Kong et al. 2006). For a more detailed description of the SINS/SINFONI sample, we refer readers to to Förster Schreiber et al. (2008). Briefly, the SINS-BzK targets are drawn directly from the list of optically selected $K \leqslant 20 \mathrm{~s}-\mathrm{BzK}$ galaxies (in the case of the Deep 3a field; Kong et al. 2006). The SINS-BX/BM sample is a fair representation of the larger Erb et al. (2006a) $\mathrm{H} \alpha$ sample in terms of source sizes and dynamical masses. However, our additional selection criteria emphasize somewhat brighter $\left(\langle F(\mathrm{H} \alpha)\rangle \sim 10^{-16}\right.$ compared to $6 \times$ $\left.10^{-17} \mathrm{erg} \mathrm{s}^{-1} \mathrm{~cm}^{-2}\right)$ and somewhat broader line width $\left(\left\langle v_{c}\right\rangle \sim\right.$ $175, \pm 68 \mathrm{~km} \mathrm{~s}^{-1}$ sample dispersion) systems than the average galaxy in the larger sample of Erb et al. (2006b, 2006c; $\left\langle v_{c}\right\rangle \sim 140$ ). For BX 482 and BzK 6004, we used SINFONI (Eisenhauer et al. 2003; Bonnet et al. 2004) on UT4 at the ESO VLT in the seeing-limited and LGSF AO modes (Rabien et al. 2003; Bonaccini Calia et al. 2006). In addition, we reanalyzed the seeing-limited $K$-data in the three BX galaxies Q2343-BX 389 $(z=2.174), \mathrm{Q} 2343-\mathrm{BX} 610(z=2.211)$ and SSA 22-MD 41 $(z=2.172)$ (Förster Schreiber et al. 2006; G. Cresci et al. 2008, in preparation). Table 1 gives the observing log and lists the FWHM angular resolutions of these data. The FWHM spectral resolution of all $K$-data cubes is about $80 \mathrm{~km} \mathrm{~s}^{-1}$. For part of our analysis we also refer to results of three additional SINS $z \sim 2$ $\mathrm{BX} / \mathrm{s}-\mathrm{BzK}$ galaxies for which we have LGSF and natural guide star (NGS) AO SINFONI observations with resolutions of $0.2^{\prime \prime}$. These galaxies are Q1623-BX $502(z=2.16$; Förster Schreiber et al. 2006), BzK 15504 ( $z=2.38$; Genzel et al. 2006), and ZC $782941(z=2.18$; SINS and COSMOS teams, in preparation). For a description of our general data reduction methods, we refer readers to Schreiber et al. (2004) and Förster Schreiber et al. (2006). With the final data cubes in hand, we smoothed the data by two spatial pixels in each spatial coordinate and then fitted Gaussian line profiles to each pixel. To blank out nonmeaningful or low signal-to-noise regions, we multiplied the resulting velocity and velocity dispersion maps with a mask constructed from the total $\mathrm{H} \alpha$ line intensity distribution.

The five main sources we discuss in this paper were selected from the SINS survey of $z \sim 1.5-3$ star-forming galaxies (Förster Schreiber et al. 2008) by two criteria. First, they are among those galaxies for which quantitative kinemetry of their velocity fields shows that they are very likely rotating disks and not undergoing major mergers (Shapiro et al. 2008). Second, among the SINS rotating disks these five galaxies are those with the highest quality data and are the best spatially resolved systems (FWHM major axis diameter $\sim 3$ to 7.5 times the FWHM instrumental resolution). This makes possible a quantitative and fairly robust analysis of the dynamics, including for the first time the determination of 
the ratio of the dynamical mass in the central few kpc to the total dynamical mass. As a result, the galaxies we have chosen belong to the largest (30th percentile) and most massive (50th percentile) members of the SINS sample, but otherwise are representative of other massive $\left(\sim 10^{11} M_{\odot}\right)$ star-forming galaxies at $z \sim 2$.

Of the three additional SINS galaxies discussed in this paper, BzK 15504 and ZC 782941 are similar to the other five large $\mathrm{BX} / \mathrm{s}-\mathrm{BzK}$ galaxies discussed above, in terms of size, mass and dynamical properties. In contrast, BX 502 is a representative of the fairly common class of compact, young, and somewhat lower mass members among the BX population in the Erb et al. (2006b, 2006c) sample, a number of which appear to be "dispersiondominated," where the random velocity dispersion is comparable to any large-scale rotational component (Law et al. 2007). For these three additional galaxies we also resolve the disk dynamics, but the data are unsuitable for a bulge analysis. The central velocity field of BzK 15504 shows an outflow potentially triggered by an AGN. The outer velocity field of ZC 782941 is disturbed by an ongoing minor merger. BX 502 is too compact for a detailed dynamical analysis.

\subsection{Modeling}

We fit the $\mathrm{H} \alpha$ velocity fields of all galaxies with simple rotating disk models (Förster Schreiber et al. 2006; G. Cresci et al. 2008 , in preparation). These disk models compute data cubes from input structural parameters. A specific model requires specifying one or several mass/light components, parameterized by azimuthally symmetric analytic functions (e.g., exponential disks, Gaussians, rings, etc.), the total dynamical mass $M_{\text {dyn }}, z$-scale height, inclination, position angle of the major axis on the sky, and a component of constant velocity dispersion throughout the disk $\left(\sigma_{01}\right)$. The model data are then convolved with the angular and spectral resolution profiles and sampled at the observed pixel scales. The total dynamical mass and mass distributions then fully define the velocity field, which is computed assuming spherical symmetry. Given the large $z$-scale heights $h_{z}$ inferred for the $z \sim 2$ galaxies (see below), this approximation holds at the $10 \%-15 \%$ level of velocity estimation (Binney \& Tremaine 2008, chapt. 2). This is sufficient for our modeling. A given $z$-scale height results in an additional $z$-velocity dispersion $\sigma_{02}$. In the approximation of a very large and very thin disk, this is given by

$$
h_{z} / R=0.5\left(\sigma_{02} / v_{d}\right)^{2}
$$

where $v_{d}(R)$ is the rotation velocity at radius $R$ (Binney \& Tremaine 2008, eq. [4.302c]). For a thick or a compact disk, equation (1) needs to be replaced by

$$
h_{z} / R=\sigma_{02} / v_{d}
$$

The total $z$-velocity dispersion in our models then is $\sigma_{0}=$ $\left(\sigma_{01}^{2}+\sigma_{02}^{2}\right)^{1 / 2}$.

To determine the model parameters that best describe each galaxy, we constructed a first-order input model with parameters for the light/mass distribution and inclination as estimated from PSF-corrected Gaussian major and minor axis FWHM sizes of the integrated $\mathrm{H} \alpha$ emission. We then refined these models iteratively to match the velocity and velocity dispersion maps for each galaxy. We determined the best-fitting galaxy parameters from $\chi^{2}$-minimization between our disk model outputs and the two-dimensional velocity and velocity dispersion maps. In the case of BX 482, we included in this $\chi^{2}$-minimization the extinction corrected, two-dimensional $\mathrm{H} \alpha$ light distribution in the op- timization (see below). For the other galaxies the $\mathrm{H} \alpha$ brightness distribution is too asymmetric and clumpy as to be usable as a detailed constraint beyond that obtained from the initial major axis size and aspect ratio above. While the disk orientation, inclination, and kinematic parameters (rotation curve, velocity dispersion distribution) can be well determined from the data sets, the details of the structural parameters (disk scale length, ring widths, size of the central mass concentration, etc.) are necessarily less well and not uniquely constrained.

The key new parameter we are able to extract from the highquality data discussed in this paper (as compared to the work of Förster Schreiber et al. 2006 and G. Cresci et al. 2008, in preparation) is a "mass concentration parameter," which is the ratio of the dynamical mass in the center (we chose $R \leqslant 0.4^{\prime \prime} \sim 3.3 \mathrm{kpc}$ ) to that in the overall observed disk to $R \leqslant 1.2^{\prime \prime}(\sim 10 \mathrm{kpc})$. This ratio can be interpreted as the mass in a central bulge or central disk to the overall disk mass. To derive this mass concentration parameter, we repeated the velocity field $\chi^{2}$-minimization along a major axis cut with the $M_{\text {dyn }}\left(\leqslant 0.4^{\prime \prime}\right) / M_{\text {dyn }}\left(1.2^{\prime \prime}\right)$ ratio as the only free variable, once the best-fitting values of inclination, positional angle, total mass, and structural parameters (ring radius and width, or disk scale length) were determined from the two-dimensional $\chi^{2}$-minimization. Along this axis the rotation velocity and velocity dispersion are maximally sensitive to the rotation curve, and thus to the mass distribution (van der Kruit $\&$ Allen 1978). This is especially true for the central regions, which are poorly resolved by our data. Our observations only provide a modest number of independent two-dimensional resolution elements across the observed galaxies. In this regime the two-dimensional velocity data off the major axis mainly serve to constrain the overall orientation of the galaxy (inclination and major axis position angle). The regions near the minor axis also are sensitive to noncircular motions, since the rotation component is minimized there (Fig. 1 of van der Kruit \& Allen 1978). For the determination of the mass concentration parameter $M_{\text {dyn }}\left(\leqslant 0.4^{\prime \prime}\right) / M_{\text {dyn }}\left(1.2^{\prime \prime}\right)$, the inclination and position angle drop out, and thus to first order do not enter the error budget. As a result, we can fit for this single parameter from the major axis data once the overall disk geometry is known.

For each galaxy we considered two types of models. The first is an exponential disk model of constant dynamical mass-to$\mathrm{H} \alpha$ light ratio and radial scale length $R_{d}$. The other is a combination of a Gaussian ring (at radius $R_{r}$ and FWHM width $2 \Delta R$ ), plus a central mass (again a Gaussian at $R=0$ and $\Delta R=0.15^{\prime \prime}-$ $\left.0.2^{\prime \prime}\right)$. Such a ring can also generically describe a flat surface brightness disk when $R_{r} \sim \Delta R$.

With some exceptions described below, the velocity and velocity dispersion fields in the five galaxies discussed here are remarkably well matched by simple disk models. The maximum residuals from the best-fit velocity fields range between 10 and $100 \mathrm{~km} \mathrm{~s}^{-1} \sim 0.05-0.4 v_{d}(\max )$. The most serious limitation of our modeling comes from the clumpiness and asymmetry of the $\mathrm{H} \alpha$ brightness distributions in most of the program galaxies, which limits the detail that can be derived for the matter distribution.

\section{RESULTS}

Figures 1-8 summarize the data, models, and data-model residuals for the five main SINS $z \sim 2$ star-forming galaxies we discuss in this paper. We also show comparisons between the integrated $\mathrm{H} \alpha$ line maps and the broadband stellar continuum maps, extracted either from the SINFONI cubes, or from the Hubble Space Telescope (HST) NICMOS/NIC2 imaging through the F160W filter ( hereafter $H_{160}$; N. M. Förster Schreiber et al. 2008, 


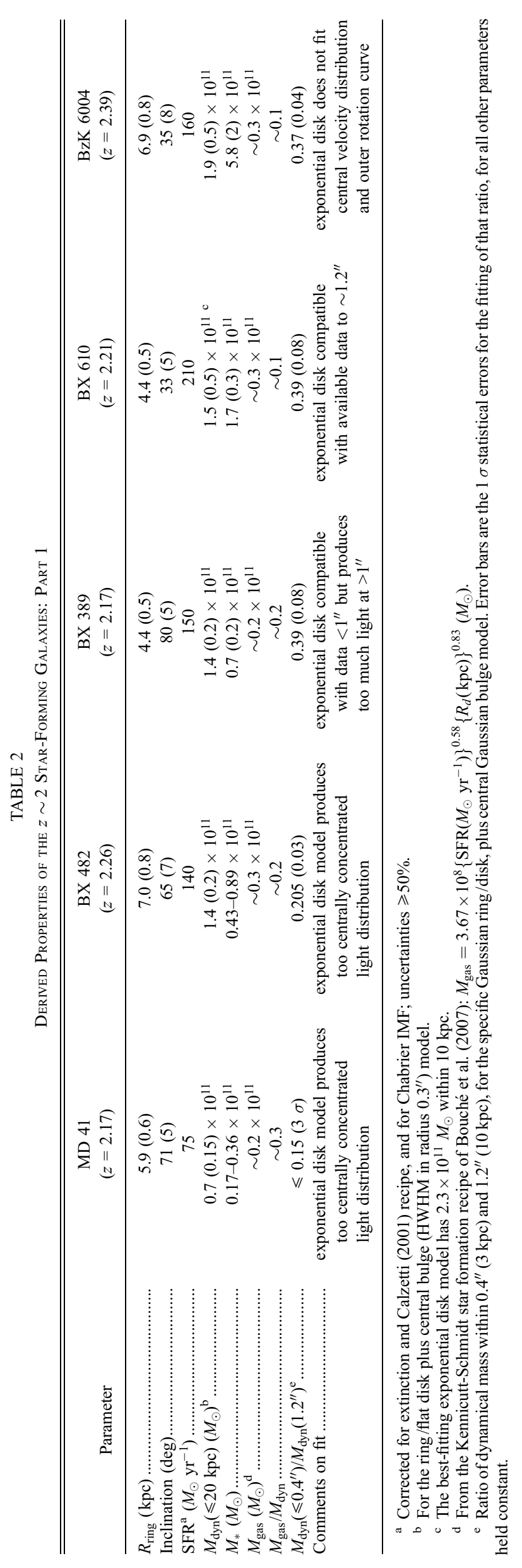


TABLE 3

Derived Properties of BX/s-BzK Galaxies: Part 2

\begin{tabular}{|c|c|c|c|c|c|c|c|c|}
\hline Parameter & BX 502 & MD 41 & BX 482 & BX 389 & ZC 782941 & BzK 15504 & BX 610 & BzK 6004 \\
\hline$v_{d}\left(\mathrm{~km} \mathrm{~s}^{-1}\right) \ldots \ldots \ldots \ldots \ldots \ldots \ldots$ & $75(25)$ & $175(30)$ & $235(40)$ & $265(40)$ & $285(30)$ & $230(30)$ & $290(70)$ & $255(40)$ \\
\hline 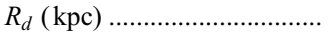 & $1.7(0.5)$ & $5.9(0.6)$ & $7(0.8)$ & $4.4(0.5)$ & $3.6(1)$ & $4.5(0.8)$ & $4.4(0.5)$ & $6.9(0.8)$ \\
\hline$\left[\mathrm{N}_{\text {II }}\right] / \mathrm{H} \alpha \ldots \ldots \ldots \ldots \ldots \ldots \ldots \ldots$ & $0.073(0.026)$ & $0.08(0.02)$ & $0.11(0.026)$ & $0.21(0.035)$ & $0.24(0.025)$ & $0.36(0.04)$ & $0.38(0.035)$ & $0.42(0.035)$ \\
\hline$M^{*} / \mathrm{SFR}(\mathrm{H} \alpha)^{\mathrm{a}}(\mathrm{Gyr}) \ldots \ldots \ldots \ldots . .$. & 0.04 & $\ldots$ & $\ldots$ & 0.5 & $\ldots$ & 0.8 & 0.8 & 3.6 \\
\hline$t^{*}(\mathrm{SED})(\mathrm{Gyr}) \ldots \ldots \ldots \ldots \ldots \ldots \ldots$ & $0.23(0.15)$ & $\ldots$ & $\ldots$ & $2.7(2)$ & $\ldots$ & $1.6\left({ }_{-1}^{+0}\right)$ & $2.7(2)$ & $2.5\left({ }_{-1.2}^{+0}\right)$ \\
\hline 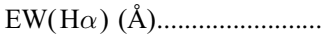 & $2200(550)$ & $>130$ & $\ldots$ & $300(75)$ & $150(38)$ & $125(30)$ & $145(36)$ & $68(17)$ \\
\hline$\Sigma_{\mathrm{SFR}}{ }^{\mathrm{a}}\left(M_{\odot} \mathrm{yr}^{-1} \mathrm{kpc}^{-2}\right) \ldots \ldots \ldots$ & 3.6 & 0.35 & 0.57 & 0.6 & 1.5 & 1.2 & 1.2 & 0.6 \\
\hline 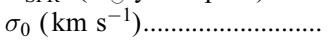 & $77(10)$ & $79(10)$ & $55(5)$ & $87(10)$ & $88(15)$ & $45(5)$ & $60(7)$ & $60(7)$ \\
\hline 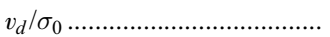 & $1(0.4)$ & $2.2(0.7)$ & $4.3(0.8)$ & $2.9(0.7)$ & $3.2(0.6)$ & $5.1(0.7)$ & $4.8(0.8)$ & $4.3(1.5)$ \\
\hline
\end{tabular}

${ }^{\text {a }}$ Uncertainties $\geqslant 50 \%$.

in preparation). Tables 2 and 3 summarize the derived physical properties of these five galaxies, and in particular the mass concentration parameter $M_{\text {dyn }}\left(\leqslant 0.4^{\prime \prime}\right) / M_{\text {dyn }}\left(1.2^{\prime \prime}\right)$ introduced in the last section. In addition to the basic structural and dynamical parameters deduced from our modeling, we also list extinctioncorrected star formation rates inferred from $\mathrm{H} \alpha$ (based on the Calzetti 2001 recipes), stellar masses inferred from modeling of the rest-frame UV/optical spectral energy distribution (see Förster Schreiber et al. 2008 for details), and gas masses estimated from the star formation rates and the universal Kennicutt-Schmidt star formation. For the latter, we adopt the gas mass-star formation relation proposed by Bouché et al. (2007). Finally we also list in Table 3 the $[\mathrm{N} \mathrm{II}] / \mathrm{H} \alpha$ flux ratio, rest-frame equivalent width of $\mathrm{H} \alpha$, and star formation surface densities.

In the next sections we first discuss the individual galaxies, starting with the new laser guide star AO observations. None of these five galaxies show evidence for an ongoing or recent major merger. Their star formation rates nevertheless are quite high. From Table 2 one can see that the time required to exhaust the present gas in these galaxies at the present star formation rate is $\sim 2 \times 10^{8} \mathrm{yr}$, an order of magnitude smaller than the interval of cosmic time $(\sim 2 \mathrm{Gyr})$ over which these galaxies are selected $(1.4<z<2.5)$, or the stellar ages determined from the SED modeling (see, however, the caveats in $\S 4.7$ ). Because of the quoted tight correlation of star formation rate with stellar mass (Daddi et al. 2007), the high star formation rates typical of these $z \sim 2$ galaxies must clearly be sustained for most of this time interval. Given that the consumption time is a factor of $\sim 10$ less, this suggests that star formation in our study galaxies is sustained by a continuous inflow of cold gas in a quasi-steady-state regime, in good agreement with the conclusions of Erb (2008).

\section{1. $Q 2343-B X 482$}

In our initial seeing-limited SINFONI H $\alpha$ data (Förster Schreiber et al. 2006), this galaxy appeared to be tadpole-shaped, with a bright emission spot to the southeast and a conical open emission region to the northwest. Our higher resolution LGSF data, as well as the NIC2 $\mathrm{H}_{160}$ image of N. M. Förster Schreiber et al. (2008, in preparation) shown in Figure 1, now reveal that the $\mathrm{H} \alpha$ and rest-frame $V$-band continuum emission form a complete, clumpy ring of radius $\sim 0.8^{\prime \prime}$, with a strong east-west asymmetry in the brightness distribution, but with a coherent kinematic structure. The additional seeing-limited data of the $5007 \AA[\mathrm{O}$ III] line exhibit an even stronger intensity asymmetry, but the same kinematics. As a result, the $[\mathrm{O}$ III $] / \mathrm{H} \alpha$ flux ratio from the seeinglimited mode for comparable resolution exhibits a smooth eastnortheast to west-southwest gradient (Fig. 1, top right panel). There is thus a large-scale gradient in either excitation/abundance or extinction. An abundance gradient is not very likely, since the $[\mathrm{N} \mathrm{II}] / \mathrm{H} \alpha$ ratio is constant across the source, to within the measurement uncertainties. If there is an east-west extinction gradient, the intrinsic $\mathrm{H} \alpha$ distribution has to be more symmetric.

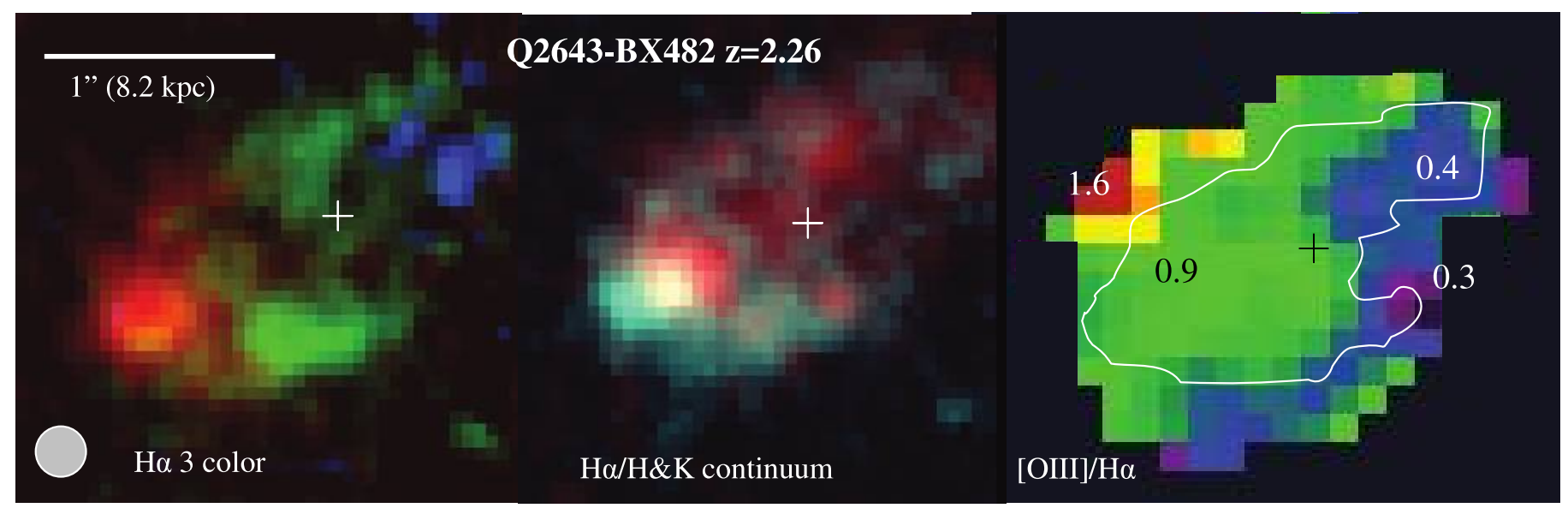

FIG. 1.- $\mathrm{H} \alpha$, [O III] line, and $H$ and $K$ continuum observations of Q2343-BX 482 ( $z=2.26$; Erb et al. 2006a, 2006b, 2006c; Förster Schreiber et al. 2006). Left: Threecolor composite of blueshifted, central, and redshifted $\mathrm{H} \alpha$ line emission, from SINFONI LGSF data at 0.2" FWHM (shaded circle). Middle: Integrated SINFONI H $\alpha$ emission (light blue) superposed on NIC2 $\mathrm{H}_{160}$ continuum (red) from N. M. Förster Schreiber et al. (2008, in preparation), with a resolution comparable to the H $\alpha$ data. The images were aligned on the brightest spot in the southeast part of the ring, whose $K$-band continuum is also detected in the SINFONI cube. The white cross marks the dynamical center of the object as determined by our modeling. Right: Observed [O III] $5007 / \mathrm{H} \alpha$ flux ratio from $0.125^{\prime \prime} \times 0.25^{\prime \prime}$ pixel, seeing-limited observations and smoothed with a $0.6^{\prime \prime}$ kernel. 


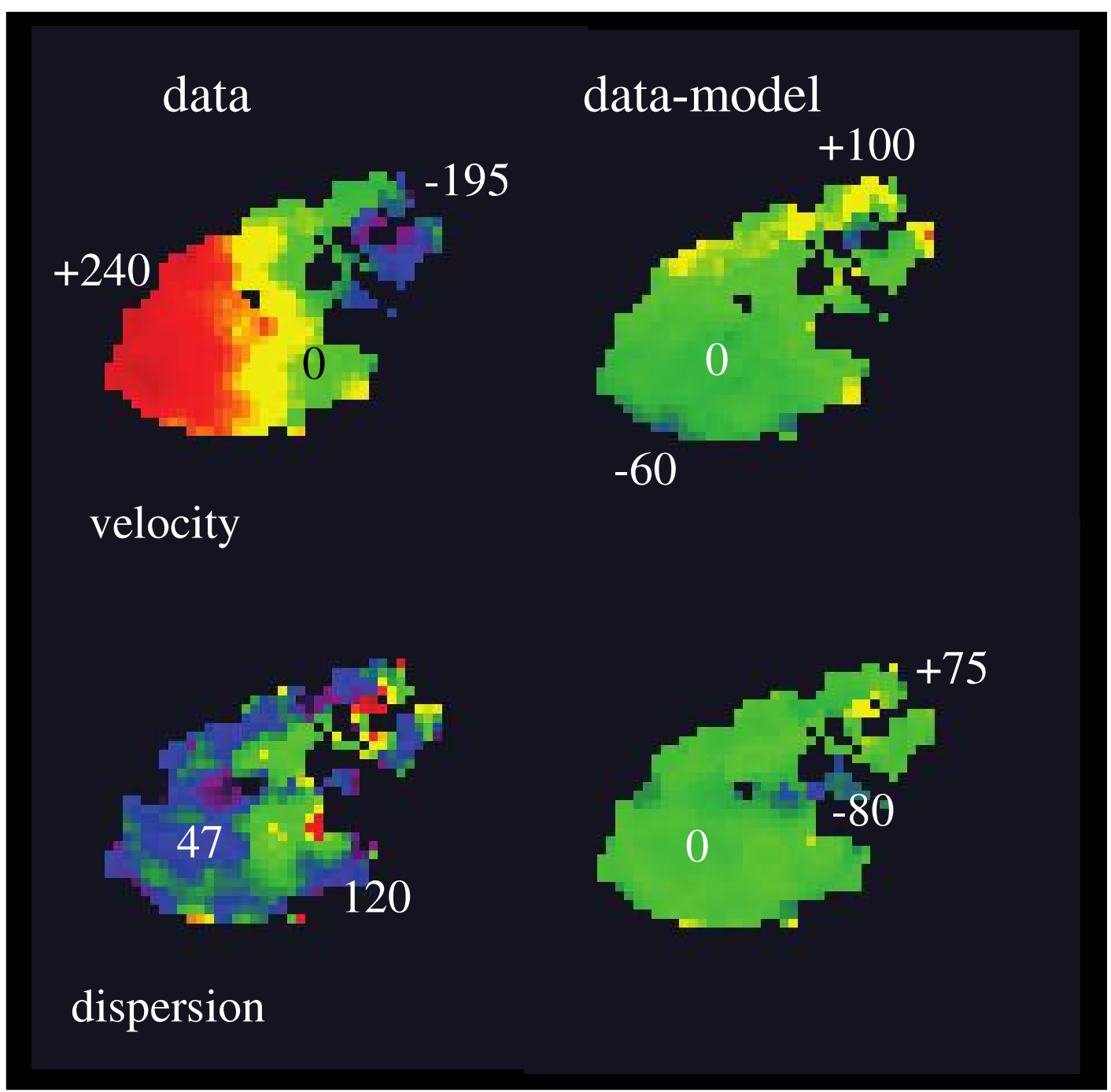

FIG. 2.-Two-dimensional $\mathrm{H} \alpha$ velocity field and kinematic modeling of BX 482. Left: Velocity centroid map (top) and velocity dispersion map (bottom). Right: Residual map (data minus model) of velocity (top ) and velocity dispersion (bottom). The model $\mathrm{H} \alpha$ emission is the best-fitting rotating ring plus bulge, as described in the text. Labels are in $\mathrm{km} \mathrm{s}^{-1}$ and denote the extreme and zero color scales.

The extinction gradient could be caused by diffuse foreground dust, or by dust partially mixed in with the $\mathrm{H} \alpha$ emission in the ring. We have used the observed $[\mathrm{O}$ III $] / \mathrm{H} \alpha$ gradient to correct for the differential extinction across the source. Adopting an LMC/SMC extinction curve and a "foreground screen" extinction model, ${ }^{14}$ the southeast to northwest asymmetry in the $\mathrm{H} \alpha$ brightness distribution disappears and in fact is reversed (top left panel of the major axis cut in Fig. 3). The extinctioncorrected two-dimensional brightness distribution is remarkably well matched by a symmetric inclined ring (inclination $65^{\circ} \pm 7^{\circ}$, position angle $-65^{\circ} \pm 5^{\circ}$ [positive $=$ east of north]). The maximum differential extinction across the entire galaxy is $\Delta A_{V} \sim 1.9$. The average residual (data - model) brightness across the galaxy is $9 \%$ of the maximum surface brightness, with a few regions deviating by up to $40 \%$.

The velocity and velocity dispersion data in Figures 2 and 3 are well fit either by a superposition of a fairly narrow $\left(2 \Delta R \sim 0.3^{\prime \prime}\right)$

14 In a screen extinction model the line intensity at wavelength $\lambda$ depends on dust opacity $\tau_{\lambda}$ at $\lambda$ as $\exp \left(-\tau_{\lambda}\right)$. For an LMC/SMC extinction curve appropriate for the subsolar abundance implied by the $\mathrm{BX} 482[\mathrm{~N} \mathrm{II]} / \mathrm{H} \alpha$ line ratio $\delta=\tau_{[\mathrm{O} \text { III }} / \tau_{\mathrm{H} \alpha} \sim 1.5$ (Calzetti 2001). The differential extinction correction to the $\mathrm{H} \alpha$ data then scales as $\{F([\mathrm{O} \text { III }]) / F(\mathrm{H} \alpha)\}^{-\beta}$, with $\beta=(\delta-1)^{-1} \sim 2$. ring at radius $R_{r}=0.85^{\prime \prime} \pm 0.1^{\prime \prime}$, plus a central mass (a bulge or central disk), or by an exponential disk model (upper right inset in Fig. 3). The former model is obviously motivated by the morphology of the $\mathrm{H} \alpha$ emission, while latter is the canonical distribution found in low- $z$ disk galaxies. For both models the maximum intrinsic rotation velocity is $v_{d}=235 \pm 40 \mathrm{~km} \mathrm{~s}^{-1}$ (middle right panel of Fig. 3). Average residuals in the velocity and velocity dispersion maps over most of the galaxy are $<10 \mathrm{~km} \mathrm{~s}^{-1}$, with larger deviations in velocity (up to $100 \mathrm{~km} \mathrm{~s}^{-1}$ ) along the northern edge of the galaxy (top right panel of Fig. 2). To match the steep slope of the major axis rotation curve and also the peak of velocity dispersion near the kinematic center (bottom left panel of Fig. 3), the ring model requires an additional central mass, either a bulge and/or a concentrated inner disk (HWHM $\sim 0.3^{\prime \prime}$ ). The best-fitting mass of the central component within $0.4^{\prime \prime}$ is $2.9 \pm 0.3 \times 10^{10} M_{\odot}$, implying a mass concentration parameter $M_{\text {dyn }}\left(\leqslant 0.4^{\prime \prime}\right) / M_{\text {dyn }}\left(1.2^{\prime \prime}\right)=0.205 \pm 0.03$. With this ring (+bulge) input model the major axis $\mathrm{H} \alpha$ intensity, velocity and velocity dispersion data are fit with $\chi_{r}^{2} \sim 1$. A ring model without a central mass component can be excluded with high significance. An exponential disk model does as well as the ring (+bulge) model in fitting the dynamical data, and implies approximately the same mass concentration parameter as the ring 

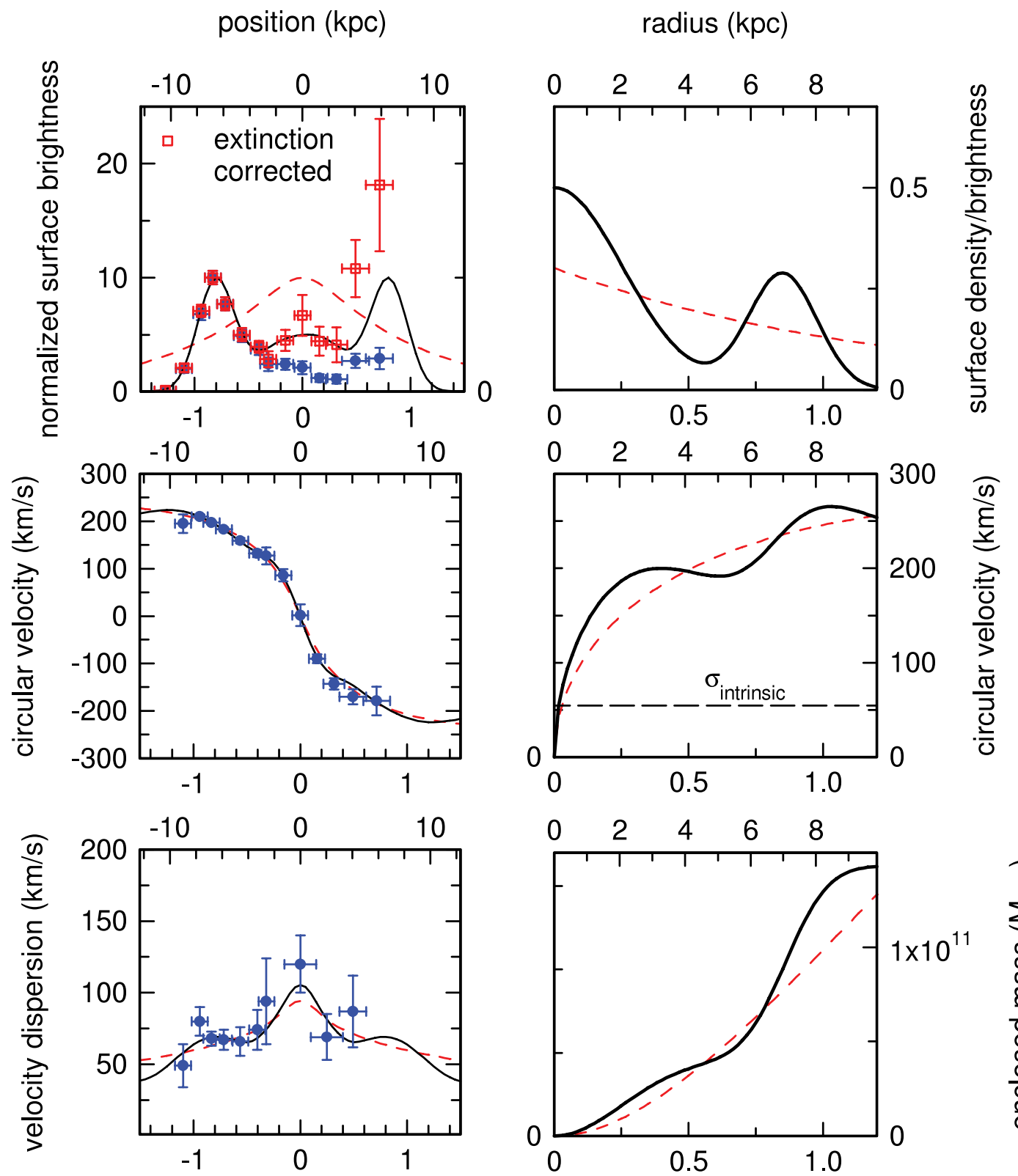

position along major axis (arcseconds)

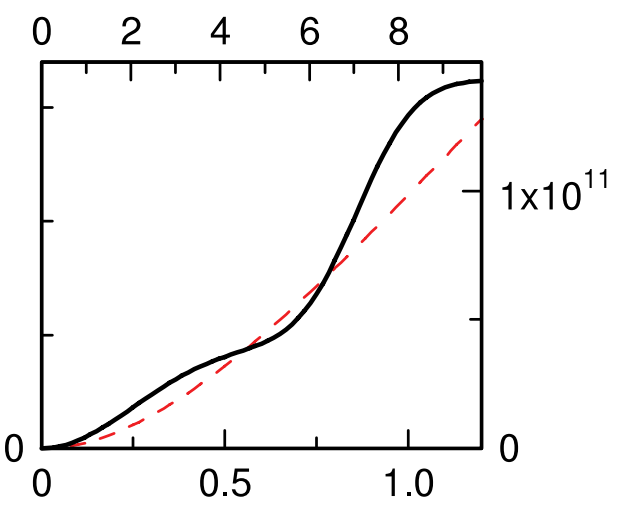

$\sum^{5}$
0
0
0
$\frac{5}{0}$
0
0
0
0
0
$\frac{0}{0}$

FIG. 3.- $\mathrm{H} \alpha$ major axis cuts and kinematic modeling of BX 482. Left: Kinematic major axis cuts in projected $\mathrm{H} \alpha$ flux (top), projected velocity (middle), and projected velocity dispersion (bottom), along P.A. $-65^{\circ}$. Blue filled circles are the measurements. Vertical-axis bars are the $\pm 1 \sigma$ errors; horizontal bars denote the aperture size. The black solid lines represent the best-fitting ring+bulge model, and the red dotted lines the best-fitting exponential disk model. The red squares in the top left panel denote the $\mathrm{H} \alpha$ fluxes corrected for screen extinction (see text). Right: Radial cuts of the best fitting, ring+bulge (solid black line) and exponential (red dotted line) model distributions for the enclosed mass (bottom), intrinsic rotation curve (middle), and mass/brightness distribution (top). The dotted horizontal line in the middle panel marks the level of the intrinsic velocity dispersion in the ring, as derived from the residual velocity distribution.

(+bulge) model ( $\sim 0.16$, bottom right inset of Fig. 3). However, the exponential disk model obviously fails to match the ring morphology of the $\mathrm{H} \alpha$ (and $H$-band continuum) maps in Figure 1. If the intensity of $\mathrm{H} \alpha$ traces the mass distribution a constant mass to light ratio, an exponential disk model is excluded with high significance in the fitting.

The obvious question is whether the ring morphology of the $\mathrm{H} \alpha$ emission may just trace the regions of currently active star formation and not the overall mass, and may thus just represent "frosting on the cake." The middle left panel of Figure 3 shows that the projected rotation velocity rises locally upward at $R \leqslant R_{r}$ and then appears to fall at $R>R_{r}$ on the eastward side. This is consistent with our assumption that the ring traces the dynamical mass. More importantly, the NIC2 image (rest-frame $V$-band) shows that the visible stellar distribution is also dominated by the ring structure (middle inset of Fig. 1; N. M. Förster Schreiber et al. 2008, in preparation). The integrated $H_{\mathrm{AB}}$-band magnitude of BX 482 is 22.4 (N. M. Förster Schreiber et al. 2008 , in preparation). Adopting a constant star formation model with $Z=0.4 Z Z_{\odot}$ tracks, a Kroupa/Chabrier IMF, and a Calzetti (2001) extinction model with $A_{V} \sim 1.5-2$, this magnitude implies a mass of live stars of $4-9 \times 10^{10} M_{\odot}$ for a plausible age range of $0.3-1$ Gyr. Adding this stellar mass and the gas mass estimated from the $\mathrm{H} \alpha$-based star formation rate and a Kennicutt-Schmidt 

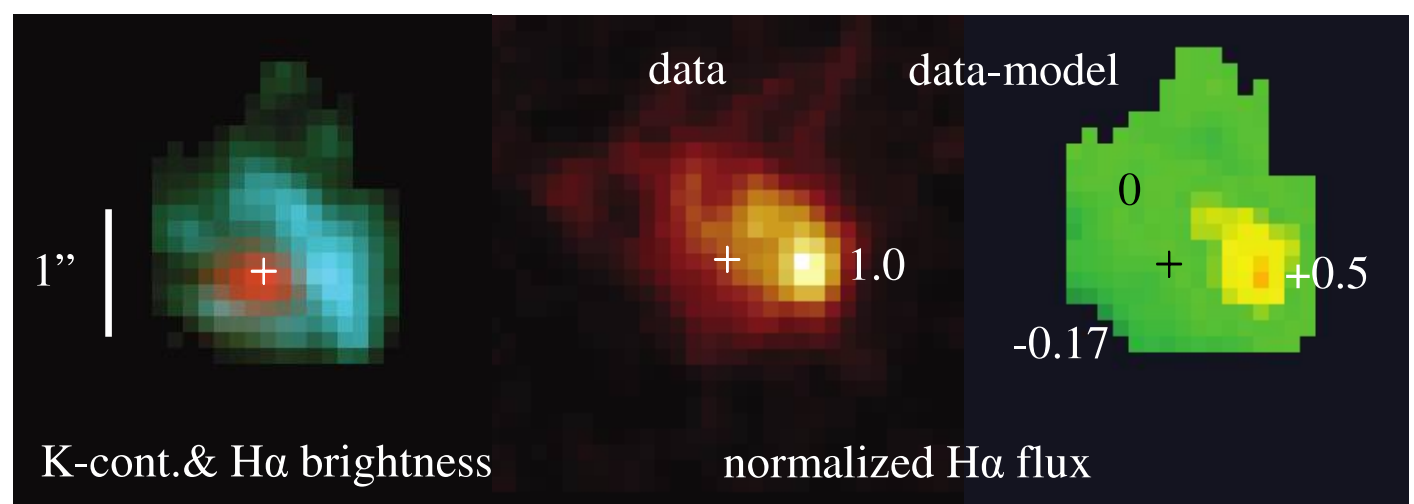

normalized Ha flux
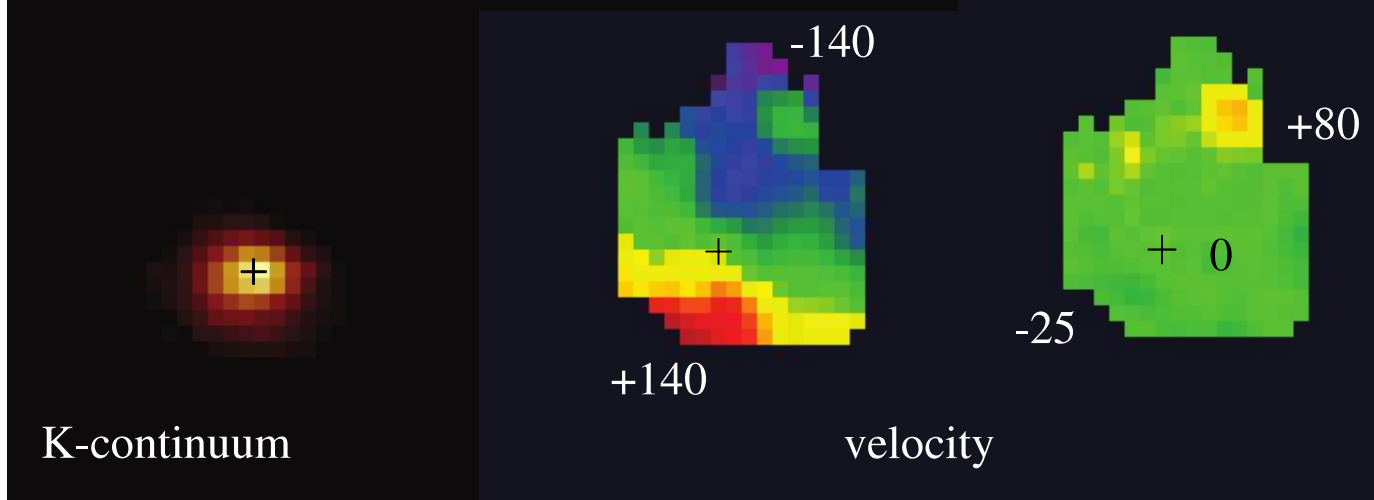

$+140$

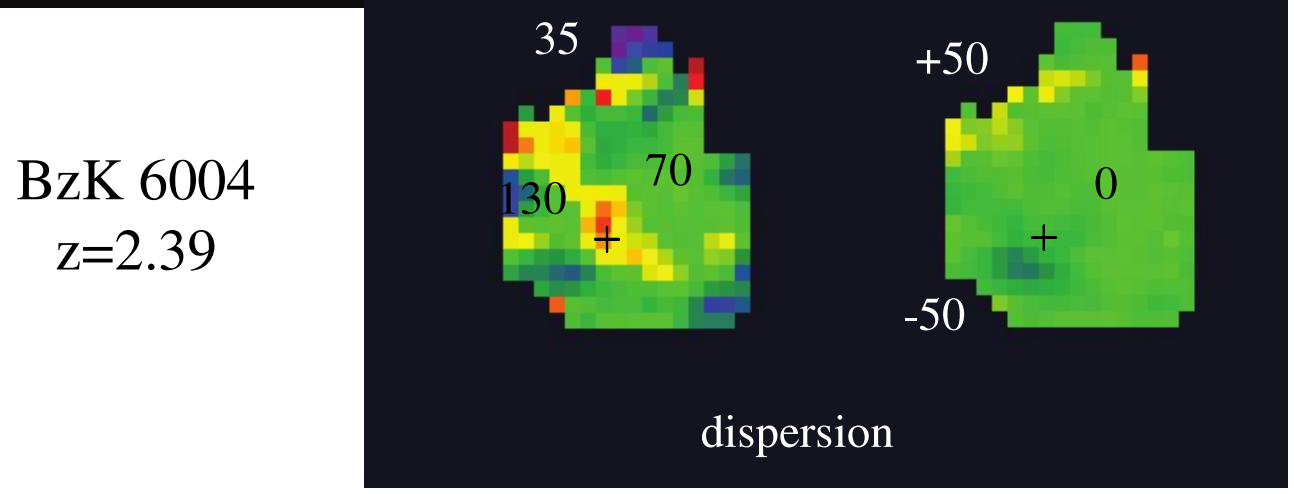

Fig. 4.-SINFONI LGSF H $\alpha$ data set of D3a6004-3482 ( $z=2.387$; Kong et al. 2006), at a resolution of 0.45" FWHM. Left: Superposition of $K$-band continuum (red) and $\mathrm{H} \alpha$ surface brightness density (peak surface brightness per wavelength interval, blue) (top) and $K$-band continuum (middle). Center: Normalized integrated H $\alpha$ emission (top), velocity map (middle), and velocity dispersion map (bottom). Right: Residual map (data minus model) of H $\alpha$ integrated emission (top), velocity (middle), and velocity dispersion (bottom). Numbers in the bottom four panels are in $\mathrm{km} \mathrm{s}^{-1}$ and denote the extreme and zero color scales.

recipe $\left(3 \times 10^{10} M_{\odot}\right.$, Table 2$)$ results in a baryonic mass of $7-12 \times 10^{10} M_{\odot}$ associated with the visible ring (+bulge) structure. Allowing for a $20 \%-40 \%$ dark matter contribution to the dynamical mass within $\sim 10-15 \mathrm{kpc}$ ( $\S 4$ below) then yields a sum of dark matter and baryonic matter of $10-18 \times 10^{10} M_{\odot}$. Our dynamical modeling yields a total dynamical mass of $14.3 \pm$ $2 \times 10^{10} M_{\odot}$, leaving little or no space for any additional underlying older stellar distribution that is not traced by our data. We conclude that the baryonic matter distribution of $\mathrm{BX} 482$ is most likely a ring, plus a small bulge/central disk.

There are significant deviations of the data from the bestfitting model. All rotating ring/disk models exhibit significant residuals in the northern part of the ring (upper right panel in Fig. 2), perhaps indicating that there is an additional largescale radial velocity component near the minor axis of the ring, with $v_{\text {radial }} \sim 85 \mathrm{~km} \mathrm{~s}^{-1} \sim 0.4 v_{d}$. This is reminiscent of the findings in BzK 15504 (Genzel et al. 2006). In that case, the radial motions may be caused by streaming along an $m=2$ (barlike) asymmetry. Furthermore, for a geometrically thin disk the re- siduals in data-model velocity dispersion are large throughout the ring. An additional random velocity component $\left(\sigma_{0} \sim 55 \pm\right.$ $5 \mathrm{~km} \mathrm{~s}^{-1}$ ) needs to be introduced everywhere in the ring. As a result, a strong conclusion of the modeling is that $v_{d} / \sigma_{0}=4.3 \pm$ 0.8 (middle panel of right column in Fig. 1). This conclusion is equivalent to the requirement in equation (1b) that the gas has a $z$-scale height of $\sim 1.6 \mathrm{kpc}$. The disk/ring of BX 482 is clearly thick and turbulent.

Table 2 summarizes the derived properties of BX 482. Unfortunately, there is no multiband photometry of this system, and we cannot determine its stellar age. Based on the approximate estimate of the stellar mass given above, the star formation age, $M_{*} / \mathrm{SFR}$, is relatively small and ranges between 0.3 and $0.6 \mathrm{Gyr}$. $\mathrm{BX} 482$ also has a low $[\mathrm{N}$ II $] / \mathrm{H} \alpha$ ratio for its dynamical mass, which in turn may imply an early evolutionary state and fairly young age ( $\S 4.1$, top right inset of Fig. 10). In morphology, BX 482 resembles the young and blue clump-cluster, tadpole, or chain galaxies in the HUDF studied by Elmegreen \& Elmegreen (2005, 2006) and Elmegreen et al. (2007). Elmegreen et al. 

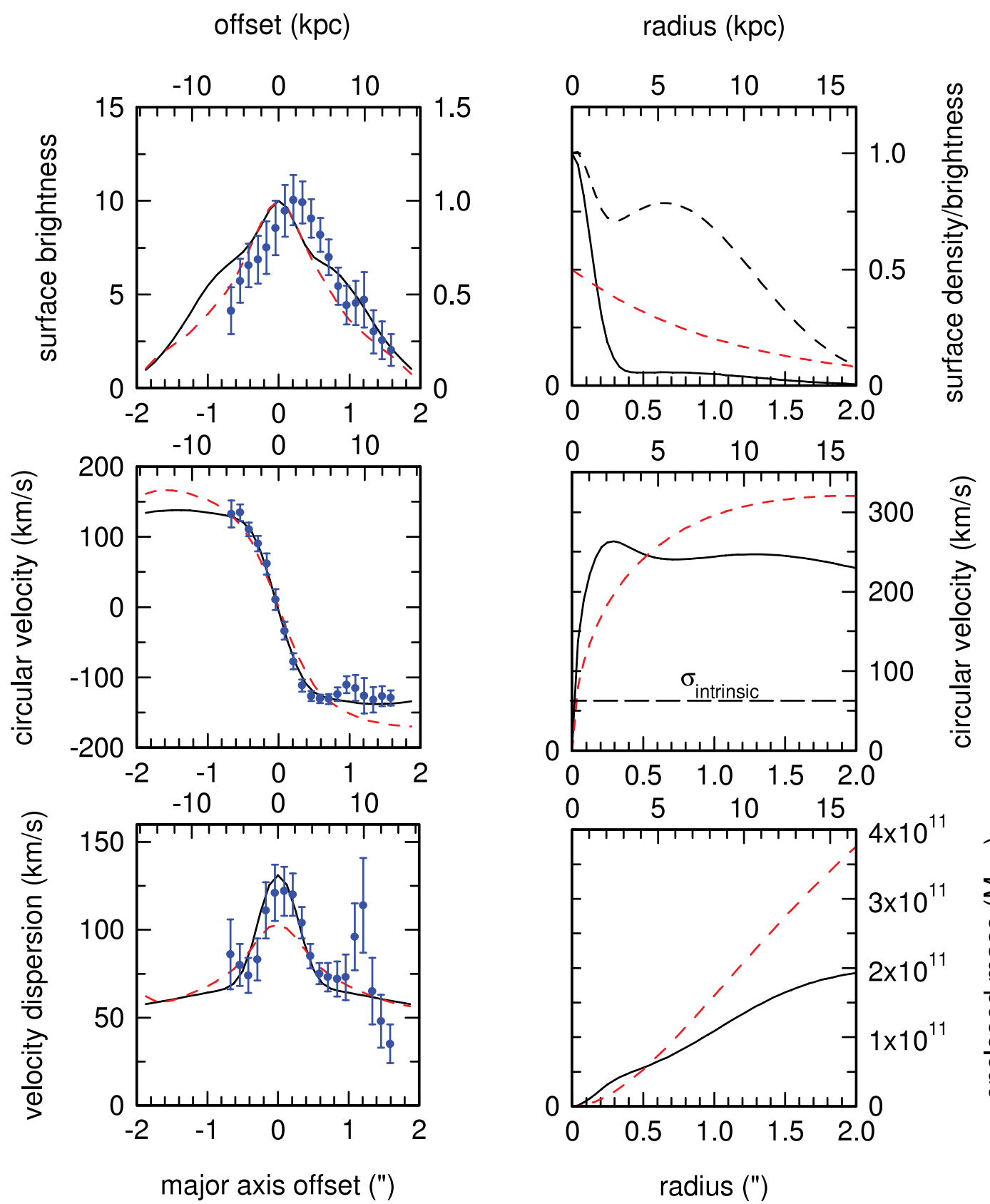

FIG. 5.- $\mathrm{H} \alpha$ major axis cuts and kinematic modeling of BzK 6004. Symbols are as in Fig. 3 for BX 482.

(2007) emphasize that a fairly large fraction of their clumpy HUDF galaxies have radial brightness distributions flatter than those of exponential disks (Sersic indices $<1$ ), again similar to that of BX 482 (Fig. 1; N. M. Förster Schreiber et al. 2008, in preparation). Their brightness distributions do not exhibit a maximum at the geometric centroid. MD 41 and BX 389, discussed below, are also examples of such chain/tadpole galaxies with flat brightness distributions.

\section{2. $B z K 6004-3482$}

The $\mathrm{H} \alpha$ emission in this fairly red and $K$-bright $\left(K_{s}=18.9\right)$ s-BzK galaxy is also well fitted by a ring or disk around a fairly massive central bulge (Figs. 4 and 5). That bulge is clearly detected in the SINFONI $K$-continuum (Fig. 4, left). Proceeding then as for BX 482 in the last section, the best-fitting model $\left(\chi_{r}^{2}=1.1\right)$ is a rotating wide ring centered at radius $R_{r}=0.85^{\prime \prime}$
$(6.9 \mathrm{kpc})$ at inclination $35^{\circ} \pm 10^{\circ}$, major axis position angle $-18^{\circ} \pm 10^{\circ}$, and maximum rotation velocity of $255 \mathrm{~km} \mathrm{~s}^{-1}$. Its large inferred width $(\Delta R \sim 7 \mathrm{kpc})$ implies that the ring effectively is identical to a flat disk (upper right panel of Fig. 5). The central bulge has a mass of $5.0 \pm 0.5 \times 10^{10} M_{\odot}$, corresponding to $M_{\mathrm{dyn}}\left(\leqslant 0.4^{\prime \prime}\right) / M_{\mathrm{dyn}}\left(1.2^{\prime \prime}\right)=0.37 \pm 0.04$. The inferred $v_{d} / \sigma_{0}$ value $(4.3 \pm 1.5)$ is quite sensitive to the adopted inclination. An exponential disk model (dotted lines in Fig. 5) is as good as the ring/disk model in terms of the $\mathrm{H} \alpha$ surface brightness distribution, but does not match the large central dispersion peak. An exponential disk model also overpredicts the rotation velocity (and thus mass) at $R>1^{\prime \prime}$ (bottom and middle left panels in Fig. 5). A combination of an exponential disk and a bulge fits the data as well as the broad ring (+bulge) model.

The derived parameters of BzK 6004 are qualitatively very similar to but more extreme than those of BX 482. The mass 
major axis offset $(\mathrm{kpc})$

radius $(\mathrm{kpc})$
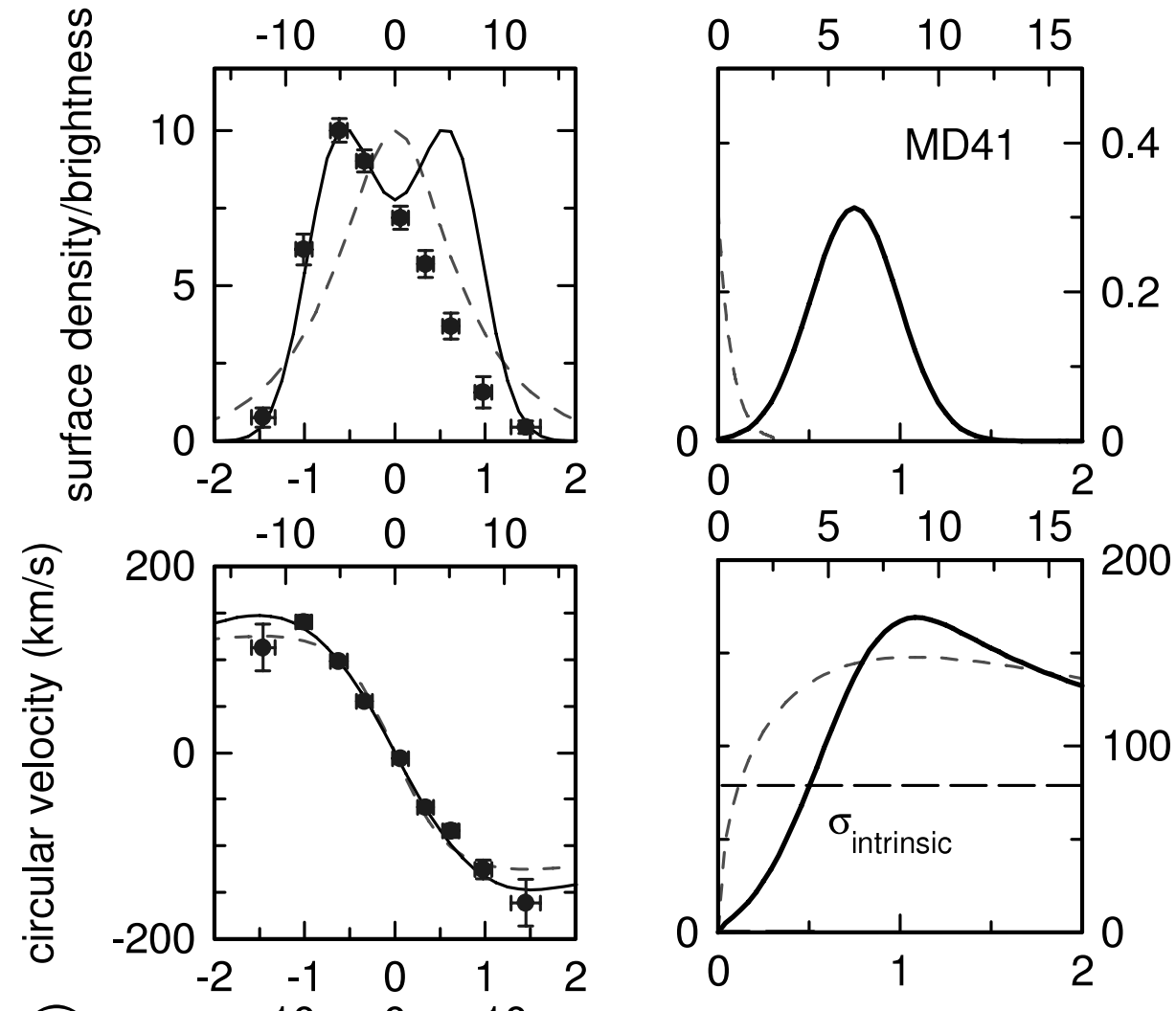

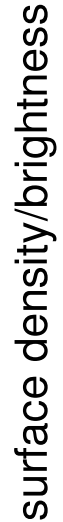
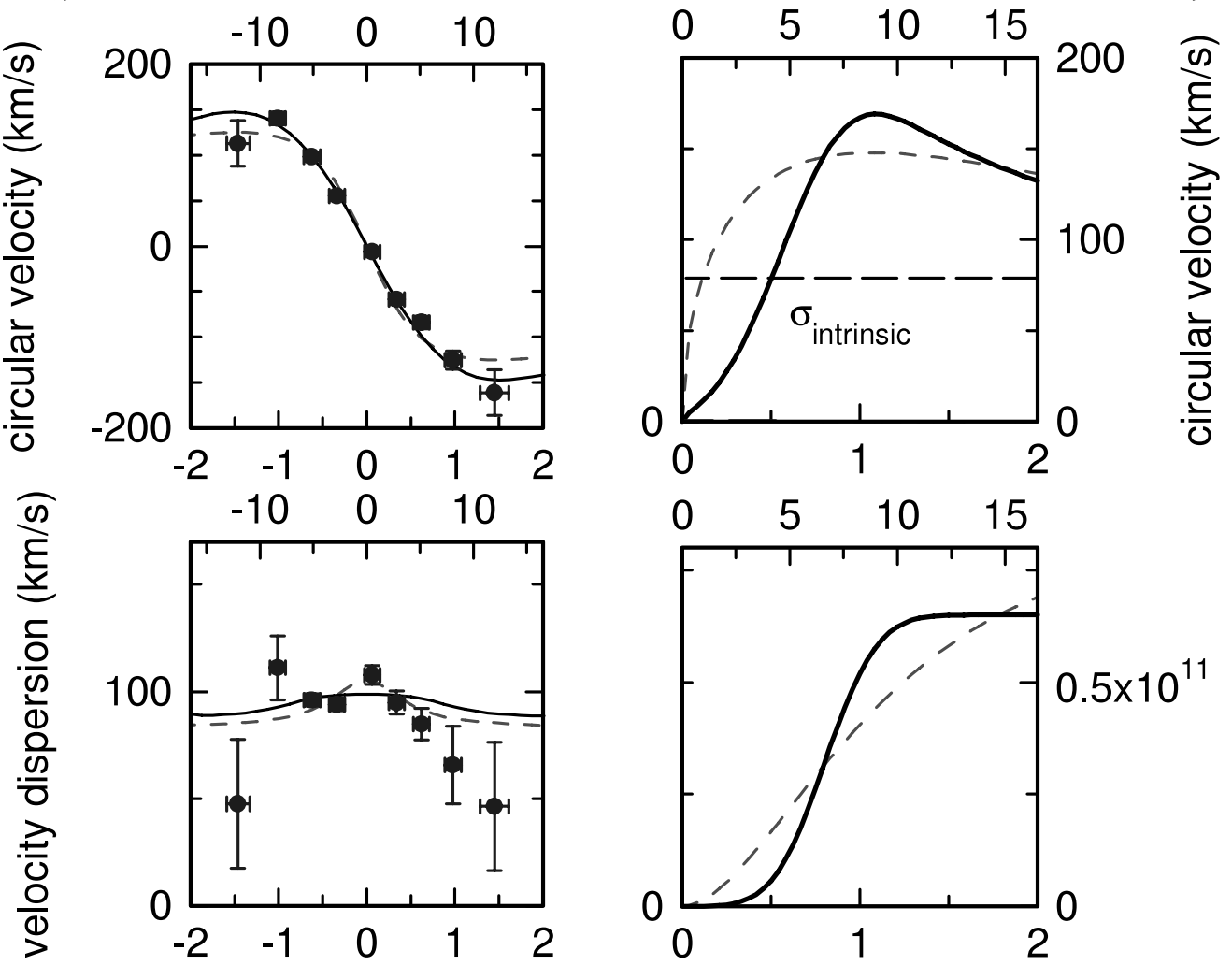

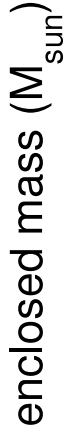

\section{major axis offset (arcseconds) radius (arcseconds)}

FIG. 6. - $\mathrm{H} \alpha$ major axis cuts and kinematic modeling of SSA $22-\mathrm{MD} 41(z=2.172)$ at a resolution of $\sim 0.5^{\prime \prime}$ FWHM (see also Förster Schreiber et al. 2006). Symbols are as in Fig. 3. [See the electronic edition of the Journal for a color version of this figure.]

concentration parameter $M_{\text {dyn }}\left(\leqslant 0.4^{\prime \prime}\right) / M_{\text {dyn }}\left(1.2^{\prime \prime}\right)$ of BzK 6004 is almost twice as large as that of BX 482. The high $K$-flux, the red color, the $\sim 2.5$ Gyr stellar age derived from population synthesis modeling of its spectral energy distribution (Förster Schreiber et al. 2008), and the large $[\mathrm{N}$ II $] / \mathrm{H} \alpha$ ratio all suggest that BzK 6004 is already quite evolved, perhaps also signaling the presence of a central active galactic nucleus (AGN). In many ways it resembles a modern Sa galaxy. Is it possible that the larger mass concentration parameter of BzK 6004 is a result of evolution?

\subsection{Q2343-BX 389, Q2343-BX 610, and SSA 22-MD 41}

For comparison to these two galaxies studied in LGSF mode, we reanalyzed three additional large BX galaxies (Q2343-BX 389, Q2343-BX 610, and SSA 22-MD 41) for which our seeing- limited data (Förster Schreiber et al. 2006) are of sufficiently high resolution to be able to place a significant constraint on the mass concentration parameter. Figures 6,7 , and 8 give the major axis cuts and best-fitting models for the three BX galaxies. These results are remarkably similar to and consistent with those in the two new sources presented above, although the poorer resolution of these data sets leads to increased uncertainties of the inferred parameters, as listed in Tables 2 and 3.

In SSA 22-MD 41, the data exhibit no significant evidence for a central mass in this high-inclination chain galaxy. The morphology of the NIC2 $H_{160}$ and ACS images (N. M. Förster Schreiber et al. 2008, in preparation) also suggests a flat extended distribution, consistent with our ring interpretation. The fairly linear rotation curve along the entire major axis of the galaxy, and the lack of a central intensity maximum or a central 


\section{major axis offset $(\mathrm{kpc})$}

radius $(\mathrm{kpc})$
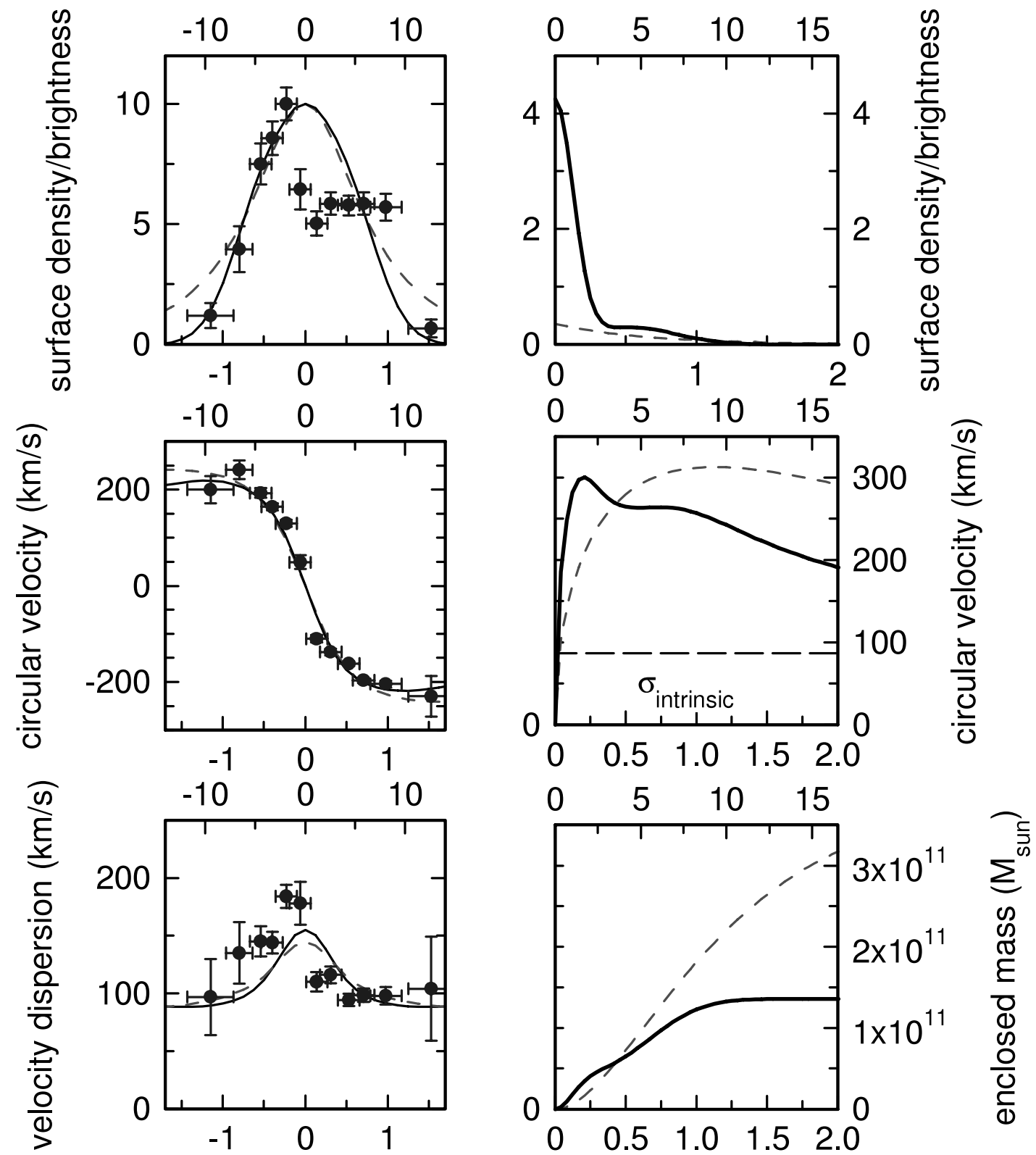

major axis offset (arcseconds) radius (arcseconds)

FIG. 7.- $\mathrm{H} \alpha$ major axis cuts and kinematic modeling of Q2343-BX $389(z=2.174)$, at a resolution of $\sim 0.5^{\prime \prime}$ FWHM (see also Förster Schreiber et al. 2006). Symbols are as in Fig. 3. [See the electronic edition of the Journal for a color version of this figure.]

velocity dispersion peak, set an upper limit of $15 \%(3 \sigma)$ to $M_{\text {dyn }}\left(\leqslant 0.4^{\prime \prime}\right) / M_{\text {dyn }}\left(1.2^{\prime \prime}\right)$. Similar to the situation in BX 482, an exponential disk model is strongly disfavored if the $\mathrm{H} \alpha$ line emission and NIC2 $\mathrm{H}$-band continuum maps trace the overall mass distribution. A constant star formation modeling with parameters similar to those adopted above for BX 482, together with the observed $H$ - and $K$-band AB magnitudes of MD 41 $\left(H_{\mathrm{AB}}=22.8, K_{\mathrm{AB}}=22.3 ;\right.$ N. M. Förster Schreiber et al. 2008, in preparation) yields a live stellar mass of $1.7-5.6 \times 10^{10} M_{\odot}$. Adding this stellar mass to the gas mass estimate of Table 3 $\left(2 \times 10^{10} M_{\odot}\right)$ is again consistent with the dynamical mass $\left(7 \times 10^{10} M_{\odot}\right)$, once allowance is made for a $20 \%-40 \%$ dark matter contribution $(\S 4)$. As in the case of BX 482, there is little or no space for any additional unseen older stellar component. An exponential disk model thus is not favored for MD 41. Likewise, the stellar mass estimate above and the extinctioncorrected $\mathrm{H} \alpha$-based star formation rate (Table 3 ) suggest a young star formation age, 0.2-0.5 Gyr.

BX 610 and BX 389, on the other hand, have 1-3 Gyr stellar/ star formation ages, as derived from multiband fitting to their spectral energy distributions (Förster Schreiber et al. 2008). They also exhibit evidence for a central mass concentration constituting about $40 \%$ of the dynamical mass in the central $10 \mathrm{kpc}$. The NIC2 $H_{160}$ image of BX 610, in particular, shows a set of bright clumps concentrated within $\sim 0.4^{\prime \prime}$ near the dynamical center of the galaxy and surrounded by a lower surface brightness disk of 


\section{major axis offset (kpc)}

\section{radius $(\mathrm{kpc})$}
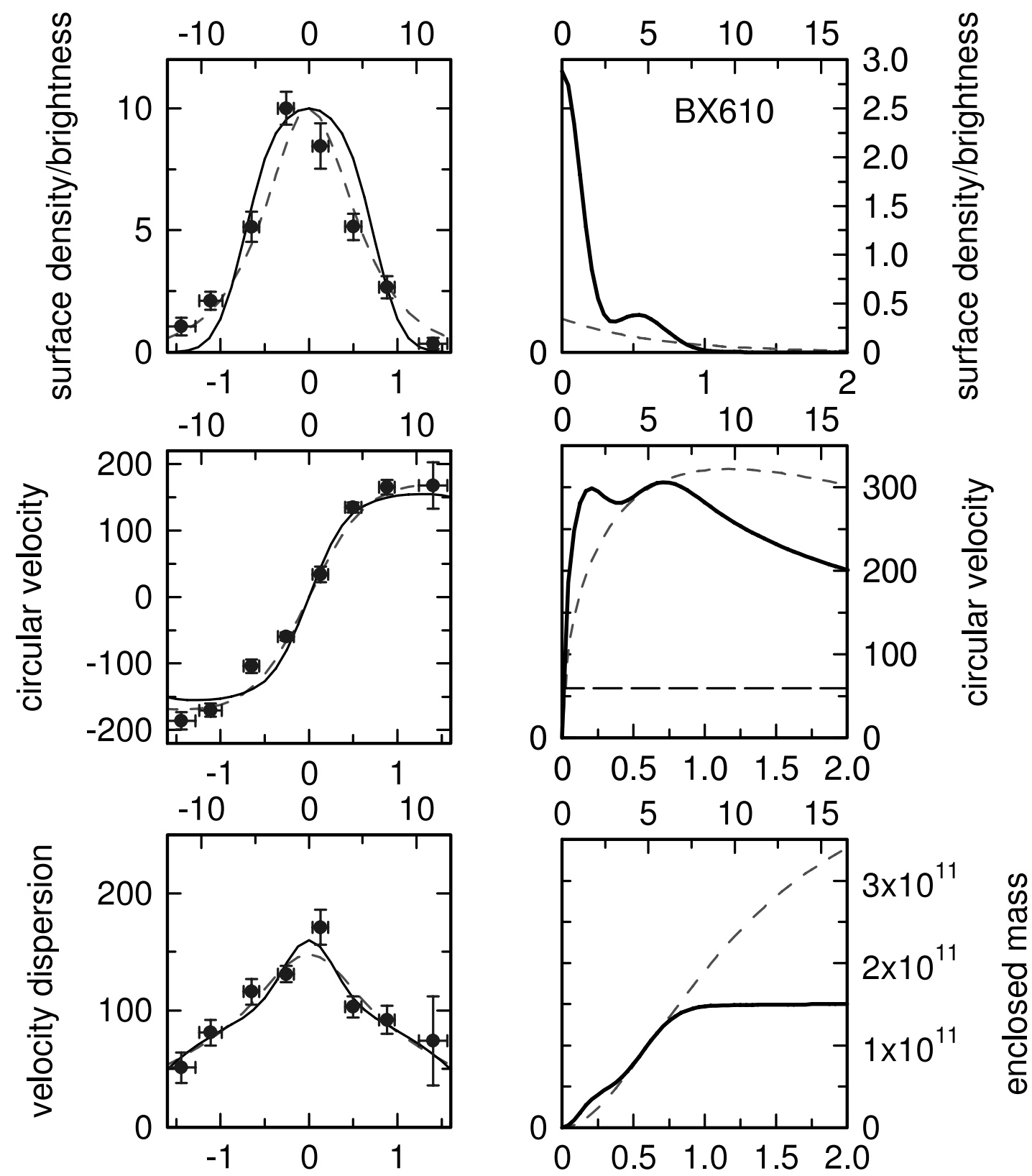

major axis offset (arcseconds) radius (arcseconds)

FIG. 8. - H $\alpha$ major axis cuts and kinematic modeling of Q2343-BX $610(z=2.211)$, at a resolution of $\sim 0.5^{\prime \prime}$ FWHM (see also Förster Schreiber et al. 2006). Symbols are as in Fig. 3. [See the electronic edition of the Journal for a color version of this figure.]

total radius $\sim 0.7^{\prime \prime}$ (N. M. Förster Schreiber et al. 2008, in preparation). For BX 389, an exponential disk (+bulge) model provides as good fits to the $R<1^{\prime \prime}$ kinematics as a wide ring (+bulge) model, but probably overestimates the mass at $R>1^{\prime \prime}$ (Fig. 7). For BX 610, an exponential disk model formally is even a somewhat better fit to the data than a ring (+bulge) model (Fig. 8).

\section{DISCUSSION}

We have shown in the last section that in the best SINS data sets we can now begin to place constraints on the dynamical mass of a central bulge/inner disk, in addition to investigating in detail the dynamical properties of the surrounding outer protodisk. In the following section we discuss the results and implications for the sources we have analyzed in 3.1-3.3. We now also include BX 502, BzK 15504, and ZC 782941 for the specific discussion of their random motions.

Essentially the entire dynamical mass in the central $0.4^{\prime \prime}$ of all sources must be baryonic. As a first-order estimate, consider a Navarro, Frenk, \& White (1997) dark matter distribution with concentration parameters of Bullock et al. (2001), with a disk angular momentum parameter of $\lambda_{d} \sim 0.1$ (characteristic for the five galaxies in Table 2; Bouché et al. 2007), and with a disk 
mass fraction (relative to the halo) of $m_{d} \geqslant \lambda_{d}$, set by the requirement of gravitational instability of the disk (Mo et al. 1998). With these assumptions, the range of maximum disk rotation velocities in Table 2 corresponds to halo circular velocities of $190 \pm 70 \mathrm{~km} \mathrm{~s}^{-1}$ and dark matter mass contribution of $10^{9.9 \pm 0.15} M_{\odot}$ within $3 \mathrm{kpc}$ of the center. This mass corresponds to $5 \%-10 \%$ of the dynamical masses we infer in Table 2 . The dark matter contributions to the total dynamical masses in Table 2 are larger. For the same assumptions as above, the dark matter mass contributions within the disk/ring radii $(\sim 6 \mathrm{kpc})$ are about $20 \%$, and within $10 \mathrm{kpc}$ they are about $40 \%$ of the total dynamical masses.

A key question is how these early central bulges/central disks formed. Are they the result of major dissipative mergers, as motivated by the CDM merger paradigm and many recent simulations (e.g., Steinmetz \& Navarro 2002)? Or have they formed through internal, secular processes, as proposed for "pseudobulges" in late-type disks at $z \sim 0$ (Kormendy \& Kennicutt 2004)? While our current galaxy sample is too small to draw detailed and robust conclusions, we show below that the data nevertheless provide interesting new and perhaps unexpected insights into this important issue. Obviously, a first hint is that none of the five galaxies in Table 2 exhibit dynamical or structural evidence for a major merger (Shapiro et al. 2008; N. M. Förster Schreiber et al. 2008, in preparation).

\subsection{Mass Concentration Parameter as a Function of Evolutionary State}

In the following section we explore the dependence of the new mass concentration parameter on other properties of the galaxies. We find that $M_{\text {dyn }}\left(\leqslant 0.4^{\prime \prime}\right) / M_{\text {dyn }}\left(1.2^{\prime \prime}\right)$ increases with evolutionary state/age.

For the five galaxies discussed in the last section, the mass concentration parameter appears to increase with the $\left[\mathrm{N}_{\text {II }}\right] / \mathrm{H} \alpha$ flux ratio (top left panel of Fig. 10). Unless the $[\mathrm{N}$ II $] / \mathrm{H} \alpha$ ratio is strongly affected by a central $\mathrm{AGN},[\mathrm{N} \mathrm{II}] / \mathrm{H} \alpha$ is a proxy of the oxygen to hydrogen abundance ratio in the ionized interstellar medium (Pettini \& Pagel 2004; Erb et al. 2006a). None of the five galaxies show kinematic evidence in our SINFONI data for a strong central AGN. The $[\mathrm{N}$ II $] / \mathrm{H} \alpha$ ratio in Table 3 refers to the value in the disk on scales of $\sim 10 \mathrm{kpc}$, which should not be strongly affected by an AGN, even if a weaker or buried AGN is present in the center. Erb et al. (2006a) have studied the massmetallicity relation at $z \sim 2$. They found that $[\mathrm{N} \mathrm{II}] / \mathrm{H} \alpha$ scales with stellar mass, the "mass-metallicity" relation. They also conclude that ". . . the $z \sim 2$ mass-metallicity relation is driven by the increase in metallicity as the gas fraction decreases through star formation. . . ." At a given mass, the metallicity would thus appear to probe the evolutionary state of a galaxy.

The upper right panel of Figure 10 shows that in our $z \sim 2$ SINS sample, $[\mathrm{N} \mathrm{II}] / \mathrm{H} \alpha$ indeed is correlated with the star formation age, $M_{*} /$ SFR (Förster Schreiber et al. 2008). This is the time required to form the current stellar mass at the current star formation rate. As a result, the mass concentration parameter $M_{\text {dyn }}\left(\leqslant 0.4^{\prime \prime}\right) / M_{\text {dyn }}\left(1.2^{\prime \prime}\right)$ also scales with star formation age. The star formation age, however, is necessarily less well determined than the $[\mathrm{N} \mathrm{II}] / \mathrm{H} \alpha$ ratio, because of the uncertainties in extinction and stellar mass. We thus prefer to show the correlation between $M_{\text {dyn }}\left(\leqslant 0.4^{\prime \prime}\right) / M_{\text {dyn }}\left(1.2^{\prime \prime}\right)$ and $[\mathrm{N}$ II] $/ \mathrm{H} \alpha$ in the top left panel of Figure 10. In further support of the correlation between metallicity and evolutionary state, three of our SINS galaxies (BX 610, BzK 6004, and BzK 6397: Förster Schreiber et al. 2006; P. Buschkamp et al. 2008, in preparation) exhibit a significant radial gradient of the $[\mathrm{N} \mathrm{II}] / \mathrm{H} \alpha$ line ratio, implying that the central regions have a $\sim 20 \%$ greater oxygen metallicity than the outer disks. The bulge regions of these three galaxies have supersolar metallicities (P. Buschkamp et al. 2008, in preparation).

Obviously, the statistical significance of a correlation based on five points is limited. Keeping this caveat in mind, our data thus suggest a scenario in which the fraction of the central bulge mass (including that of the surrounding central disk) appears to increase secularly over timescales of less then a few Gyr.

\subsection{Turbulent and Clumpy Disks}

The eight actively star-forming galaxies in Table $3(\sim 0.2-$ $3 M_{\odot} \mathrm{yr}^{-1} \mathrm{kpc}^{-2}$ ) all have a large component of random local gas motion, $\sigma_{0} \sim 45-90 \mathrm{~km} \mathrm{~s}^{-1}$, in agreement with the earlier finding of Förster Schreiber et al. (2006). Given the spatial and spectral properties of the other $\sim 50$ galaxies in the SINS survey, this conclusion appears to hold generally for the entire population of $z \sim 2$ star-forming galaxies studied so far by integral field spectroscopy (Förster Schreiber et al. 2006, 2008; Wright et al. 2007; Law et al. 2007). The ratio $v_{d} / \sigma_{0}$ ranges from 2 to 5 in the seven large disk galaxies, as compared to $10-20$ in $z \sim 0$ disks (e.g., Dib et al. 2006). Yet smaller values of $v_{d} / \sigma_{0} \sim 1$ are found for a number of the compact BX galaxies, such as BX 502 (Table 3; Law et al. 2007 and in preparation; Förster Schreiber et al. 2008). These latter systems appear to be gas rich and dispersion-dominated. The $z \sim 2 \mathrm{BX} / \mathrm{s}-\mathrm{BzK}$ galaxies in the SINS survey are turbulent and geometrically thick $\left(h_{z} \sim 1 \mathrm{kpc}\right)$.

The high-resolution $\mathrm{H} \alpha$ and $H / K$ images of the $\mathrm{BX} / \mathrm{s}-\mathrm{BzK}$ galaxies, as well as the rest-frame UV-continuum images in most other high-redshift star-forming galaxies, are typically dominated by a modest number (5-10) of bright, compact clumps (Cowie et al. 1995; van den Bergh et al. 1996; Elmegreen \& Elmegreen 2005, 2006; Elmegreen et al. 2007; N. M. Förster Schreiber et al. 2008 , in preparation). In many cases these giant star-forming complexes make up a significant fraction $(\sim 20 \%-40 \%)$ of the mass of the disk (Elmegreen \& Elmegreen 2005; Elmegreen et al. 2007; Genzel et al. 2006). The SINFONI AO data sets for BX 482 and BzK 15504, as well as the NIC2/ACS images of BX 482, BX 389, BX 610, and MD 41, have sufficiently high resolution for determining the intrinsic clump sizes. Their FWHM diameters, $L_{c}$, range from $0.15^{\prime \prime}$ to $0.45^{\prime \prime}$ (Fig. 9). In BX 482 (where both NIC2 and $\mathrm{H} \alpha$ data are available), stellar and gas clump sizes are comparable. Elmegreen \& Elmegreen (2005, 2006) find similar results for the clump-cluster/chain galaxies in the HUDF, on the basis of photometric analysis of rest-frame UV/ACS images. In their study of 10 clump-cluster galaxies at $\left\langle z_{\text {phot }}\right\rangle \sim 2.3$, Elmegreen \& Elmegreen (2005) infer an average clump size of $L_{c}=0.22^{\prime \prime}$. The HUDF galaxies are somewhat lower luminosity/mass analogs of the galaxies we are studying here $\left(\left\langle M_{\text {gal }}\right\rangle \sim 10^{10.7} M_{\odot},\left\langle\mathrm{SFR}_{\text {gal }}\right\rangle \sim 20 M_{\odot} \mathrm{yr}^{-1},\left\langle v_{d}\right\rangle \sim\right.$ $150 \mathrm{~km} \mathrm{~s}^{-1},\left\langle M_{c}\right\rangle \sim 10^{8.8} M_{\odot}$; Elmegreen \& Elmegreen 2005$)$. These galaxies appear to dominate the $z>1$ cosmic star formation in the HUDF (Elmegreen et al. 2007).

The Jeans length in a gravitationally unstable gas disk with $Q_{\text {gas,Toomre }} \leqslant 1$ is

$$
L_{\mathrm{Jeans}}=\frac{\pi}{\sqrt{2}}\left(\frac{\sigma_{0}}{v_{d}}\right) R_{r} Q_{\mathrm{Toomre}}
$$

which yields $\left\langle L_{\text {Jeans }}\right\rangle \sim 2.5 \mathrm{kpc}$ or $0.3^{\prime \prime}$ for the parameters appropriate for the galaxies in Tables 2 and 3. The observed sizes of the giant $\mathrm{H} \alpha$ clumps/star forming complexes in the clumpy $z \sim 2$ galaxies are consistent with the Jeans lengths inferred from 
size $(\mathrm{kpc})$

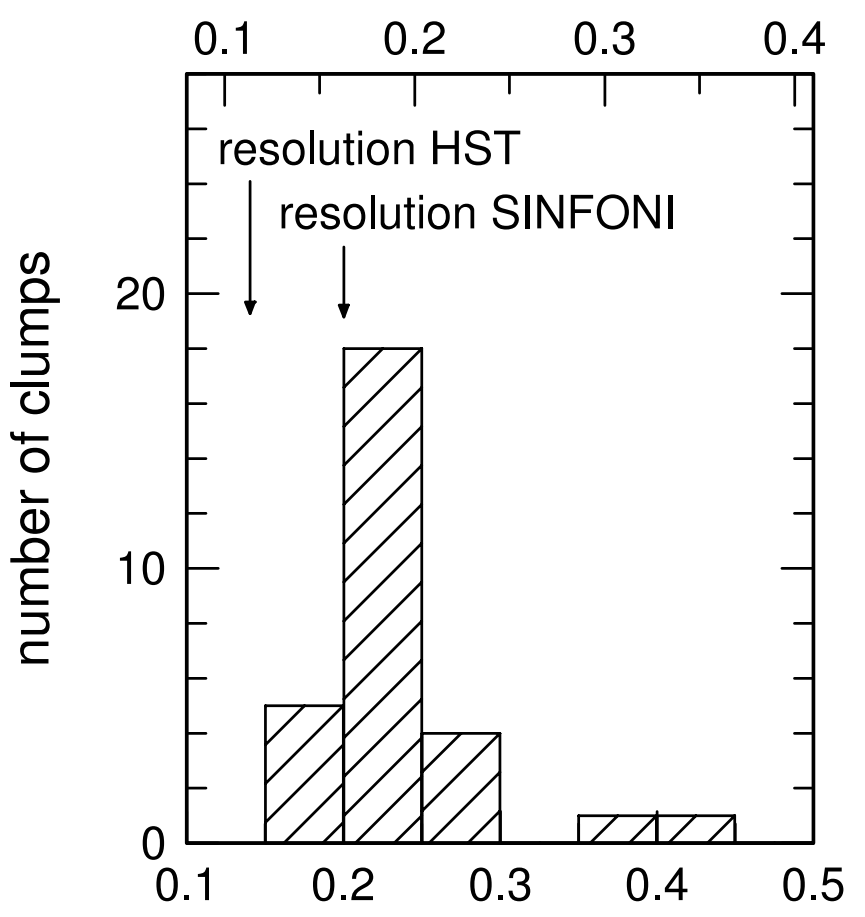

FWHM size (arcseconds)

FIG. 9.-Distribution of observed FWHM sizes of the star-forming clumps in massive star-forming $z \sim 2$ galaxies. Sizes were determined by Gaussian fits to 29 fairly isolated clumps in the SINFONI data of BzK 15504 (Genzel et al. 2006) and BX 482 (resolution 0.2"), as well as the $\mathrm{NIC} 2 \mathrm{H}_{160}$ data of BX 389, BX 610, and MD 41 (N. M. Förster Schreiber et al. 2008, in preparation).

the galaxies' kinematics properties. The H $\alpha$ clumps thus may be initially close to virial equilibrium. Given their large sizes and the overall large surface densities of the galaxy disks (a few $10^{2} M_{\odot} \mathrm{pc}^{-2}$ ), as derived from our dynamical measurements, it is clear that the star formation complexes must be very massive (see also Elmegreen \& Elmegreen 2005),

$$
M_{c}=\frac{L_{c}^{2} v_{d}^{2}}{4 G R_{d}} \sim 10^{9.4 \pm 0.5} M_{\odot} .
$$

These masses are obviously much larger than even the most massive $\mathrm{H}$ II regions in $z \sim 0$ starburst galaxies $\left(10^{7}-10^{8} M_{\odot}\right)$.

In the standard picture of disk formation from gas accretion from the halo (e.g., Mo et al. 1998), the radius of the initial starforming disk is set by the characteristic angular momentum parameter $\lambda_{h}\left(\left\langle\lambda_{h}\right\rangle \sim 0.04\right.$; Bett et al. 2007) of the accreting gas, $R_{d} \sim \lambda_{h} R_{\mathrm{vir}} / \sqrt{2}$, where $R_{\mathrm{vir}}(\sim 100-150 \mathrm{kpc}$; Förster Schreiber et al. 2006) is the virial radius of the dark matter halo in which the disk resides. This scenario is in good agreement with the observed sizes of the BX/s-BzK disks in the SINS sample, provided that the baryonic angular momentum is conserved during the accretion and that the larger systems we are discussing here are associated with halos of fairly high $\left(\lambda_{h} \sim 0.1\right)$ angular momentum parameters (Bouché et al. 2007) or, alternatively, that the incoming baryonic gas creating the proto-disks has a larger angular momentum than the average dark matter. Such high baryonic angular momentum parameters may be the result of the specific formation paths of their halos (L. V. Sales et al. 2008, in preparation).

\subsection{Secular Evolution is Efficient at High Redshift}

In a smooth gaseous disk, the timescale for radial secular evolution is given by the viscous drag time (Shakura \& Sunyaev 1973; Silk \& Norman 1981; Lin \& Pringle 1987; Silk 2001),

$$
t_{\mathrm{visc}}=R^{2} / \eta \text { for viscosity } \eta,
$$

such that for $\eta=\alpha h_{z} \sigma_{0}$ (Shakura $\&$ Sunyaev 1973) with $z$-scale height $h_{z}$, dimensionless parameter $\alpha \approx 1$, and disk rotation velocity $v_{d}=\left(R / h_{z}\right) \sigma_{0}$,

$$
t_{\mathrm{visc}}=\frac{R^{2}}{\alpha h_{z} \sigma_{0}}=\frac{1}{\alpha}\left(\frac{v_{d}}{\sigma_{0}}\right)^{2}\left(\frac{R}{v_{d}}\right)=\frac{1}{\alpha}\left(\frac{v_{d}}{\sigma_{0}}\right)^{2} t_{\mathrm{dyn}}(R) .
$$

Once fragmentation and star formation sets in $\left(Q_{\text {gas }} \leqslant 1\right.$, eq. [2]), the secular evolution timescale is then given by the dynamical friction timescale of the clumps against the background of the disk/halo. Based on Chandrasekhar's formula (Noguchi 1999; Silk 2001; Immeli et al. 2004a, 2004b; Binney \& Tremaine 2008), the dynamical friction timescale is

$$
t_{\mathrm{df}}=\beta\left(\frac{R}{\lambda_{\mathrm{Jeans}}}\right)^{2} t_{\mathrm{dyn}}(R)=\beta\left(\frac{v_{d}}{\sigma_{0}}\right)^{2} t_{\mathrm{dyn}}(R),
$$

with an (uncertain) dimensionless factor $\beta \sim 0.3$ (see above references). Here $t_{\text {dyn }}$ is the dynamical timescale, $t_{\text {dyn }}=2.4 \times$ $10^{7} R_{6} / v_{250} \mathrm{yr}, R_{6}$ is the disk radius in units of $6 \mathrm{kpc}$, and $v_{250}$ is the disk circular velocity in units of $250 \mathrm{~km} \mathrm{~s}^{-1}$. Other secular processes in a stellar disk, such as bar formation and transport, occur on a timescale $t_{\mathrm{bar}} \sim 30-50 t_{\mathrm{dyn}}\left(R_{6}\right)$ (Immeli et al. $2004 \mathrm{a}$, 2004b; Bournaud \& Combes 2002). Disk galaxies at $z \sim 0$ have $v_{d} / \sigma_{0} \sim 10-20$ (e.g., Dib et al. 2006) and secular processes proceed on a timescale of several Gyr or more (Kormendy \& Kennicutt 2004). The $z \sim 2$ star- forming galaxies we study here are clearly different. In these galaxies, $v_{d} / \sigma_{0}$ ranges between 1 and 5. Compared to $z \sim 0$, gaseous and stellar processes proceed faster by at least one order of magnitude or more,

$$
\begin{aligned}
t_{\mathrm{sec}}(z \sim 2) & \sim 10-30 t_{\mathrm{dyn}}(R) \sim 0.5 \mathrm{Gyr} \\
& \sim t_{\mathrm{dyn}}\left(R_{\mathrm{vir}}\right) \sim 0.2 t_{H}(z \sim 2) .
\end{aligned}
$$

Here $t_{\text {dyn }}\left(R_{\text {vir }}\right)$ is the dynamical time for crossing the virial radius of the dark matter halo. Exactly which secular process dominates depends on the stellar and gas fraction, whether $Q_{\text {gas }}$ is greater or less than $Q_{\text {stars }}$ (Immeli et al. 2004a, 2004b), and whether bar instabilities occur in a turbulent environment.

We propose that in the five cases we have presented in this paper, we are witnessing the rapid buildup of central bulges through such secular processes. Our observations are in excellent agreement with the numerical simulation work of Noguchi (1999), Immeli et al. (2004a, 2004b), and Bournaud et al. (2007), which we discuss further in $\S 4.5$.

Secular evolution of disks has been previously considered for the origin of the so-called pseudobulges of $z \sim 0$ late-type spiral galaxies (Kormendy \& Kennicutt 2004), growing over a timescale that is comparable to the present Hubble time. Such a long timescale appears to be at variance with the uniformly old age of stars in the bulge of the Milky Way (Zoccali et al. 2003), suggesting formation by early merging rather than late disk instability. Our finding of a very short timescale for the disk instability in $z \sim 2$ disks reopens this issue, making it quite plausible 

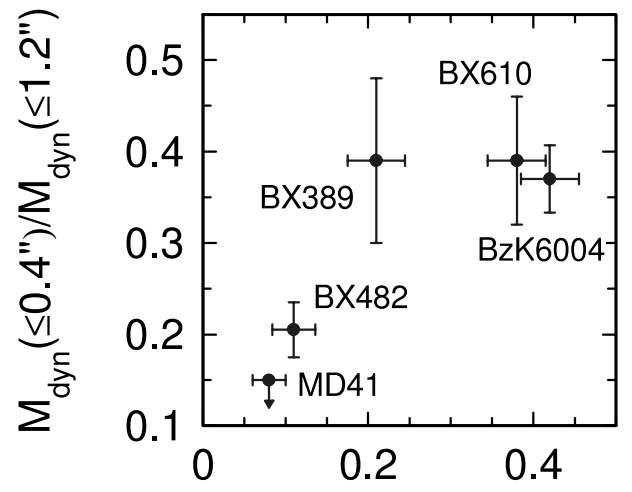

$[\mathrm{NII}] / \mathrm{H} \alpha$

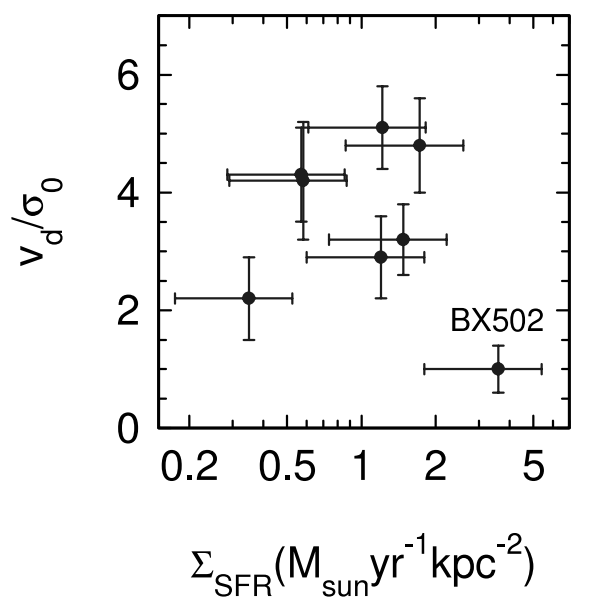

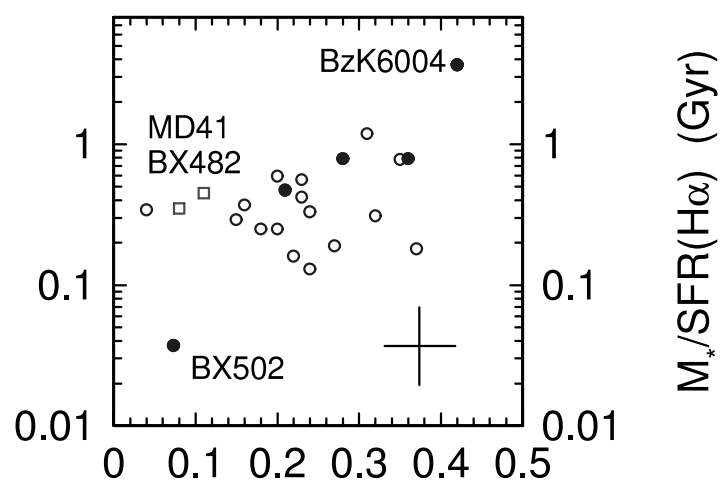

$[\mathrm{NII}] \mathrm{H} \alpha$

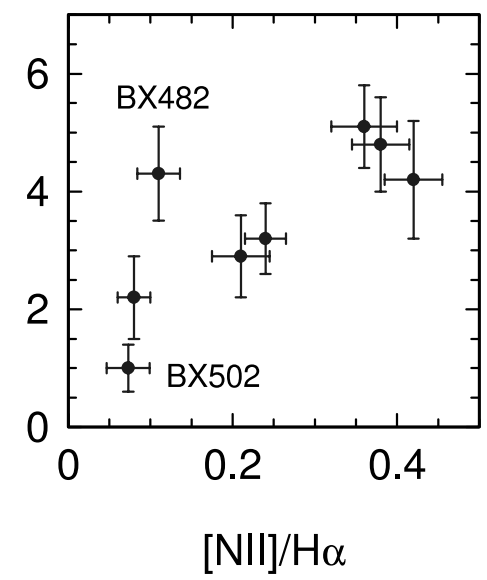

FIG. 10.- Parameter correlations. Top left: Derived ratio of dynamical masses within $0.4^{\prime \prime}(\sim 3.3 \mathrm{kpc})$ and $1.2^{\prime \prime}(\sim 10 \mathrm{kpc})$ for the five optically/UV-selected star-

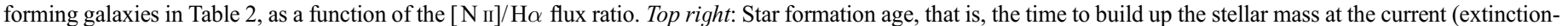

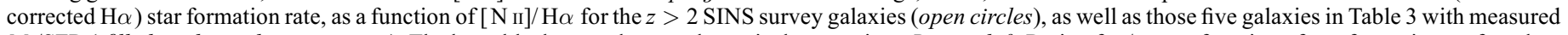

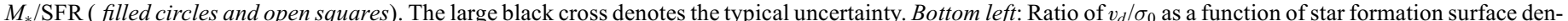

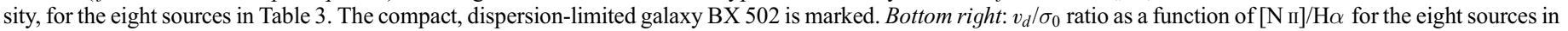
Table 3. [See the electronic edition of the Journal for a color version of this figure.]

that the Galactic bulge resulted from fast disk instability some 10 Gyr ago. In support of this conjecture, Melendez et al. (2008) have found that the $\alpha /$ Fe element abundances of the old Galactic bulge are more or less identical to those of the thick disk, implying that these two components are coeval and both formed rapidly.

\subsection{Large Turbulent Velocities Driven by Cold Flows}

It is not obvious what causes the large velocity dispersions and large implied scale heights of the high- $z$ disks. Here it is important to realize that the rest-frame UV/optical continuum data of our sample galaxies, as well as the HUDF galaxies of Elmegreen $\&$ Elmegreen $(2005,2006)$, imply the same $\sim 1 \mathrm{kpc} z$-scale heights in the stars as in the ionized gas traced by $\mathrm{H} \alpha$. The large turbulence is probably a property of the entire star-forming gas and stellar layer, and not just a feature of the ionized gas. One obvious possibility is that these motions are the result of feedback from the intense star formation itself, through the combined action of winds, supernova explosions, and radiation transport (Efstathiou 2000; Silk 2001; Thompson et al. 2005). Theoretical estimates and extrapolations from local starburst galaxies (Monaco 2004; Dib et al. 2006) make a plausible case that the observed velocity dispersions $\left(45-90 \mathrm{~km} \mathrm{~s}^{-1}\right.$ ) can be reached at the ob- served star formation surface densities in the $\mathrm{BX} / \mathrm{s}-\mathrm{BzK}$ galaxies. In this case, one would expect that the $v_{d} / \sigma_{0}$ ratio scales inversely with the star formation rate surface density $\Sigma_{\text {SFR }}$. The bottom left panel of Figure 10 is a first attempt at testing this assertion. There is no obvious correlation between the observed values of $v_{d} / \sigma_{0}$ and $\Sigma_{\mathrm{SFR}}$ among the seven massive galaxies in Table 3. The turbulence in the compact, young and high star forming surface density system BX 502 is much greater than in the other galaxies. Including this system may add some evidence that feedback may indeed be at work in the systems with the largest star formation surface densities. BX 502 has a much lower mass than the other seven galaxies, and it is not yet clear how these compact dispersion-limited systems relate to the massive large disks and whether they have experienced a recent merger (Law et al. 2007).

A second possibility is that the turbulence is driven by the accreting gas as it enters the forming disk. Recent high-resolution adaptive mesh simulations by Ocvirk et al. (2008) clearly demonstrate that in the cold flow regime (Dekel \& Birnboim 2006; Keres et al. 2005), gas is brought in radially along the filaments of the cosmic web, from well outside the virial radius of the halo and all the way to the disk region. It would appear to be unavoidable that the rapidly inflowing $\left(v_{\text {inflow }}=\gamma v_{d}\right.$, with a 
dimensionless number $\gamma \sim 1-1.4$ ) gas streamers and clumps (minor mergers?) embedded in the cold flows shock and stir up the forming protodisk (e.g., Abadi et al. 2003). There are no realistic simulations yet of the disk/flow interaction and shocks. The rate of specific energy gain is $\dot{E}_{\mathrm{acc}}=\beta \gamma^{2} v_{d}^{2} / t_{\mathrm{acc}}(z)$, where $t_{\mathrm{acc}}=\left\langle M_{\mathrm{gas}} /\left(d M_{\mathrm{acc}} / d t\right)\right\rangle_{z}$ is the gas accretion time at $z$. If the rate of turbulent energy loss of the disk clumps is driven by their physical collision rate $\dot{E}_{\text {coll }}=-\sigma_{0}^{2} / t_{\text {coll }}$, the velocity dispersion is given by (Förster Schreiber et al. 2006)

$$
\sigma_{0} \sim \beta \gamma^{2} v_{d} \sqrt{t_{\mathrm{coll}} / t_{\mathrm{acc}}}
$$

For the parameters of the observed disks with 5-10 virialized clumps of radius $\sim 1 \mathrm{kpc}$, the collisional timescale is close to the dynamical timescale of the ring/disk, $t_{\text {coll }}=\varepsilon t_{\text {dyn }}$ with $\varepsilon \sim 1$. From a statistical analysis of the Millenium dark matter simulation (Springel et al. 2005), Genel et al. (2008; see also Birnboim et al. 2007) derive a fitting formula for the average accretion timescale of dark matter as a function of $z(t)$ and halo mass $M_{h}$,

$\left\langle t_{\mathrm{acc}}\left(z, M_{h}\right)\right\rangle=2.27 \times 10^{9}\left(\frac{1+z}{3.2}\right)^{-2.2}\left(\frac{M_{h}}{2 \times 10^{12} M_{\odot}}\right)^{-0.08} \mathrm{yr}$.

For the cosmic baryon fraction of $\sim 0.2$, the baryonic accretion rates implied by equation (9) would also be sufficient to explain sustained star formation rates of $>100 M_{\odot} \mathrm{yr}^{-1}$ in the massive $\mathrm{BX} / \mathrm{s}-\mathrm{BzK}$ galaxies in this paper. Equations (8) and (9) then yield

$$
\begin{aligned}
v_{d} / \sigma_{0} \sim & 9.7 \beta^{-1} \gamma^{-2}\left(v_{250} R_{6}^{-1} \varepsilon^{-1}\right)^{1 / 2} \\
& \times[(1+z) / 3.2]^{-1.1} M_{2 \mathrm{E} 12}^{-0.04}
\end{aligned}
$$

where $M_{2 \mathrm{E} 12}$ is the halo mass in units of $2 \times 10^{12} M_{\odot}$. These numbers are appropriate for the massive $\mathrm{BX} / \mathrm{s}-\mathrm{BzK}$ galaxies we consider here. The simple estimate in equation (10) suggests that the conversion of accretion energy may account for the observed turbulent motions if the product $\beta \gamma^{2} \varepsilon^{1 / 2}$ exceeds $\sim 2$. Equation (10) also indicates that over 2-3 Gyr of cosmic evolution (from $z \sim 4$ to $z \sim 2$ ), the average $v_{d} / \sigma$ ratio would be expected to increase by a factor of 1.75 , from the cosmological decrease of the accretion rate. A still larger decrease in the accretion rate would be predicted once the halos grow above the hot/cold flow boundary (Dekel \& Birnboim 2006; Ocvirk et al. 2008) and if AGN feedback is effective. Stirring of the disk turbulence by the accretion flows would thus naturally predict that an early phase of rapid accretion, leading to clumpy, thick protodisks and secular bulge formation, is followed a few Gyr timescale later by a less violent regrowth of disks, perhaps related to the formation of thin disks.

The bottom right panel in Figure 10 is an attempt to explore whether there is a trend of $v_{d} / \sigma_{0}$ with evolutionary state, again as above in form of the $[\mathrm{N} \mathrm{II}] / \mathrm{H} \alpha$ ratio. With the exception of BX 482, there may indeed be a trend for the more mature sources to also be the ones with the smallest relative fraction of turbulent motion (largest $v_{d} / \sigma_{0}$ ). As with the other trends discussed in this section, a larger sample of well-resolved kinematic data sets will be required to make more definite statements.

The scenario we are discussing relies on the assumption that the accreting material is highly gas-rich. A simple check of this assertion is possible if one assumes that below a certain critical halo mass, supernova feedback prevents early star formation from converting a large fraction of the baryonic gas into stars (Dekel \& Silk 1986; Efstathiou 2000). Following Dekel \& Silk (1986) and Dekel \& Woo (2003), this critical baryonic mass is given by

$$
M_{b, \text { crit }}=2.0 \times 10^{10}\left(\frac{v_{d, \text { crit }}}{100 \mathrm{~km} \mathrm{~s}^{-1}}\right)^{3}\left(\frac{1+z}{3.2}\right)^{-2 / 3},
$$

where we have again assumed a baryon fraction in the disk of $b \sim 0.2$. Halos with masses below $M_{b \text {, crit }} / b$ form stars inefficiently and should be gas rich. This critical mass is a factor of 3.5-10 smaller than the dynamical/stellar masses of the five galaxies in Table 2. Given that the galaxies we have observed appear not to be undergoing major mergers, any minor merger accretion would have to have a mass ratio $>3: 1$. Such minor mergers would be at or below $M_{\text {crit }}$, and thus would be very gasrich according to equation (11). Here we presume that in the limit of very minor mergers (mass ratios $\gg 10: 1$ ), smooth gas accretion and a series of minor mergers are virtually identical.

We conclude from this section that if the trends suggested by the present data are confirmed, the level of turbulence in the high- $z$ disks may be strongly influenced by (the evolution of) the accretion rate, as well as stellar feedback in the most intensely star-forming systems. The large observed turbulence of the high- $z$ disks may be plausibly understood with current estimates of cold-flow gas accretion rates based on dark matter simulations.

\subsection{Comparison to Simulations}

Stimulated initially by the clumpy appearance of high-z galaxies on HST rest-frame UV-images (chain galaxies; Cowie et al. 1995; van den Bergh et al. 1996) and later by the work of Elmegreen \& Elmegreen (2005, 2006), several groups have carried out numerical simulations of the evolution of gas-rich, fragmentation unstable, "clumpy" galaxies with star formation recipes (Noguchi 1999; Semelin \& Combes 2002; Immeli et al. 2004a, 200b; Bournaud et al. 2007). These simulations vary in technical approach ( $N$-body simulations vs. hydro-grid code) and initial assumptions. For instance, Bournaud et al. (2007) adopt an ab initio unstable disk, while Semelin \& Combes (2002) start with hot gas that then becomes gravitationally unstable after cooling to the molecular phase. Noguchi (1999) and Immeli et al. (2004a, 2004b) let the disk to grow to instability from rapid gas inflow along the polar axis. The latter authors start with a pure gas disk in a dark matter halo. Bournaud et al. introduce, in addition, an initial stellar component making up 50\% of the mass. While Bournaud et al. assume that the initial stellar disk is already geometrically thick but do not include stellar (supernova) feedback, Immeli et al. consider supernova feedback with a two-phase interstellar medium. Keeping in mind these differences, as well as the simplifications that none of these simulations begin from self-consistent cosmological initial conditions, all these studies find essentially the same salient features:

1. Rapidly forming, very gas-rich disks will become violently and globally unstable to fragmentation into giant star forming clumps if the Toomre instability parameter in the gas layer suddenly becomes $Q_{g} \leqslant 1$ across much of the entire disk. This requires that the (cold) gas accretion rate is high $\left(>100 M_{\odot} \mathrm{yr}^{-1}\right)$ and happens on a timescale comparable to the orbital timescale of the 
initial disk ( $\sim 0.5$ Gyr in the simulations of Noguchi 1999 and Immeli et al. 2004a, 2004b).

2. Once fragmentation sets in, the disk evolves rapidly during a short-lived (0.4-1 Gyr) "clump phase." During this phase, star formation proceeds rapidly in the giant clumps, with a star formation rate comparable to the gas accretion rate. The strong dynamical interactions heat the gas and stellar components, with a resulting $z$-scale height of $\sim 1 \mathrm{kpc}$.

3. As a result of efficient dynamical friction of the clumps against the background of the rest of the disk, the clumps spiral into the center and form a central bulge and a surrounding smooth exponential stellar disk. Clump-clump collisions dissipate the large turbulent motions.

Samland \& Gerhard (2003) considered a case with a much lower accretion rate $\left(\sim 20-40 M_{\odot} \mathrm{yr}^{-1}\right)$. In this model, the disk grows from inside out, with a bulge forming first from low angular momentum gas inflow. The surrounding disk is quite thick, and the most active star formation occurs in a ring that moves over time outward from the center. The reason for outward propagation is that the gas infall can no longer compensate for the gas consumption by star formation in the center. This in turn is caused by the decrease in gas accretion to the central regions due to increasing specific angular momentum of the infalling material. The ring grows in radius until eventually the disk becomes unstable and forms a bar.

One prediction of these simulations and the generic hypothesis of clump evolution from smooth accretion of gas from the halo is that the clumps would plausibly have similar metallicities, while the interclump gas would be expected to have much lower metallicities. This is indeed consistent with our current data, to within the limitations of signal-to-noise ratio and resolution. With the exception of radial $[\mathrm{N} \mathrm{II}] / \mathrm{H} \alpha$ gradients mentioned above, there are no indications for significant $[\mathrm{N} \mathrm{II]} / \mathrm{H} \alpha$ variations from clump to clump in the disk regions of the five galaxies of our main sample, nor in the three additional larger systems in Table 3. The signal-to-noise ratio is not yet sufficient to explore the interclump metallicity.

It is remarkable how well the observed properties of the young disks we study in this paper, the simple "back of the envelope" considerations presented above, and the more detailed simulations appear to be consistent with each other. The interesting consequence and prediction of these simulations is that these clumpy, turbulent disk galaxies are going through a transitory "fragmentation" phase at the very beginning of their evolution.

\subsection{Comments on Asymmetries}

In this paper and in our previous papers (e.g., Förster Schreiber et al. 2006; Genzel et al. 2006), we have emphasized the surprisingly symmetric properties of the kinematics of the optical/ UV-selected massive star-forming galaxies we have observed. This is an obvious (and intended) simplification. Asymmetries of the brightness and velocity distributions are present in essentially all the SINS data sets (this paper; Förster Schreiber et al. 2006, 2008; Genzel et al. 2006). As discussed in the section on BX 482 (§ 3.1), local variations in extinction, excitation, and local current star formation events may account for some of these asymmetries, even if the underlying kinematics and potential are intrinsically symmetric. However, there are clearly are also intrinsic variations in the kinematic properties that can be seen from asymmetric rotation curves (e.g., Figs. 7 and 8) and from residuals in the velocity distributions (e.g., Figs. 2 and 4) after subtraction of a first-order rotation model (see Genzel et al.
2006, G. Cresci et al. 2008, in preparation, and Förster Schreiber et al. 2008, in preparation, for more examples). These features may be a natural consequence of asymmetries in the underlying potential and gas accretion processes. Lopsided rotation curves as observed in a number of the SINS galaxies have also been seen in the $\mathrm{H}_{\mathrm{I}}$ kinematics of some $z \sim 0$ galaxies (Swaters et al. 1999) and are ascribed there to long-lived intrinsic $m=1$ modes in the underlying potential and to interactions with nearby galaxies. At high redshift, strong asymmetries would be naturally be expected to be caused by the nonisotropic and filamentary accretion, by the large radial velocities of the accreting gas, and by the minor merger activity (e.g., Ocvirk et al. 2008). In the framework of the gas-rich, clumpy galaxies, very significant local perturbations from a smooth rotation curves are expected as a result of the large clump masses (Immeli et al. 2004a, 2004b; Bournaud et al. 2007).

\subsection{Consequences for Galaxy Evolution}

We have presented evidence that massive bulges and spheroids (with masses up to at least $5 \times 10^{10} M_{\odot}$ ) may have formed on a timescale of 1-3 Gyr through secular evolution from gasrich, turbulent disks. The upper end of this mass range would be consistent with bulge masses for $z \sim 0$ Sa bulges. These turbulent disks grow from steady and rapid gas accretion (including minor mergers) from the dark matter halos and fragment into very large star formation clumps. Unless major mergers happen during this phase, it would appear that most galaxies experience such a clump phase, which is an inevitable consequence of the combination of rapid continuous gas accretion, large gas fraction, and large turbulence. This conclusion is consistent with the large fraction of such clumpy galaxies in the HUDF (Elmegreen et al. 2007) and the strong correlation between stellar mass and star formation in the $z \sim 2 \mathrm{UV}$ - and optically selected galaxies (Erb et al. 2006c; Daddi et al. 2007). In addition to rapid bulge buildup, this process would also account rather easily for the fact that there are no descendent massive, thick disks at $z \sim 0$ (Förster Schreiber et al. 2006). Estimates based on the Millenium simulation suggest that the major merger rate is too low to destroy all $M_{*} \sim 10^{11} M_{\odot}$ galaxies between $z \sim 2$ and 0 (Genel et al. 2008; Fakhouri \& Ma 2008; Conroy et al. 2008). It is tempting to speculate that the thick, old stellar disks seen in the Milky Way and nearby galaxies (e.g., Freeman \& Bland-Hawthorn 2002; Yoachim \& Dalcanton 2006) are the remnants of this $z \sim 2-3$ phase of transient evolution of clumpy, turbulent disks fed by rapid cold flows.

Efficient secular evolution may also help to explain the remarkably efficient and rapid star formation in the $z \sim 2$ starforming galaxy population (Förster Schreiber et al. 2006; Genzel et al. 2006; Daddi et al. 2007). Davé (2008) has introduced the star formation activity parameter

$$
\alpha_{\mathrm{SF}}(z)=\frac{M_{*}}{\operatorname{SFR}\left(t_{H}(z)-1 \mathrm{Gyr}\right)},
$$

where $\alpha_{\mathrm{SF}}$ is equal to unity if the stellar formation timescale $M_{*} / \mathrm{SFR}$ is just equal to the available cosmic (Hubble) time at $z$, taking into account that most of the star formation occurred after reionization $(<1 \mathrm{Gyr})$. Figure 11 shows that most of the observed $z \sim 2$ star-forming s-BzK and BX galaxies, including the majority of the SINS survey galaxies (N. M. Förster Schreiber et al. 2008, in preparation) and the five galaxies in Table 3 with a determination of $M_{*}$, appear to have formed their 

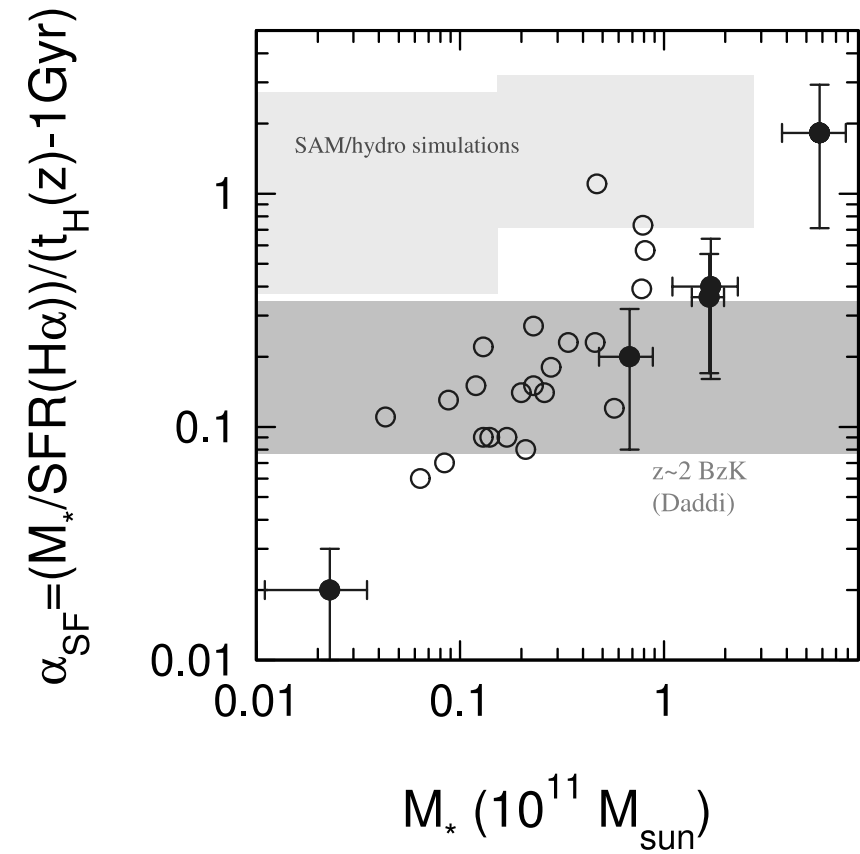

FIG. 11.-Star formation activity parameter for $z \sim 2$ star-forming galaxies. The gray-shaded region is the observed galaxies in the s-BzK survey of Daddi et al. (2007), and green shaded region marks the predictions from current semianalytic and hydrodynamic galaxy formation simulations as shown in Davé (2008). The best-quality SINS galaxies from Förster Schreiber et al. (2008) are shown by open circles, while filled circles (with $1 \sigma$ uncertainties) show those five galaxies in Table 3 with a determination of $M_{*}$. Most of the observed $z \sim 2$ starforming galaxies appear to be forming stars at a rate faster than the available Hubble time, in contrast to the predictions from simulations. The trend of $\alpha_{\mathrm{SF}}$ with mass for the SINS galaxies is largely a result of the $\mathrm{H} \alpha$ luminosity threshold in the sample (Förster Schreiber et al. 2008). [See the electronic edition of the Journal for a color version of this figure.]

stars on a timescale a few times faster than the available Hubble time (see also Daddi et al. 2007). This is in disagreement with the simulations for which $\alpha_{\mathrm{SF}} \sim 1$ (Davé 2008; Kitzbichler $\&$ White 2007).

Secular evolution, together with efficient star formation at high gas surface densities, may explain why the star formation activity parameter $\alpha_{\mathrm{SF}}$ is significantly less than 1 at high redshift. For a Schmidt-Kennicutt star formation recipe, the star formation rate surface density scales approximately inversely proportional to the dynamical timescale (Kennicutt 1998; Bouché et al. 2007). If the gas in the initial disks fragments into compact dense clumps and at the same time the overall disk contracts as a result of the dynamical friction process, the star formation activity parameter would naturally decrease by a factor of several. Furthermore, Bouché et al. (2007) have presented evidence that the star formation efficiency per dynamical timescale in high-density starforming galaxies, such as the $z \sim 2 \mathrm{BX} / \mathrm{s}-\mathrm{BzKs}$, is about 0.07 , or 3 times larger than that estimated for normal $z \sim 0$ spirals (Kennicutt 1998). For this purpose, gas masses were inferred from $\mathrm{CO}$ rotational line luminosities calibrated at the low gas surface density end by the normal $z \sim 0$ star-forming galaxies from Kennicutt (1998). On the high end, the calibration is by $z \sim 0$ ultraluminous infrared galaxies (ULIRGs; Kennicutt 1998) and $z \sim 1-3$ SMGs (Tacconi et al. 2006, 2008). For the normal galaxies, Bouché et al. (2007) used a Milky Way CO-to$\mathrm{H}_{2}$ conversion factor (as did Kennicutt 1998), while for ULIRGs and SMGs Bouché et al. applied a CO-to- $\mathrm{H}_{2}$ conversion factor $\sim 0.2$ times that of the Milky Way (see Tacconi et al. 2008 for a more detailed discussion). For the gas surface densities derived in this way for the BX/s-BzK galaxies, the Bouché et al. (2007) fit gives the following expression for the $\alpha_{\mathrm{SF}}$ parameter:

$$
\alpha_{\mathrm{SF}}(z \sim 2)=0.24\left(\frac{\Sigma_{\mathrm{gas}} / \tau_{\mathrm{dyn}}}{20 M_{\odot} \mathrm{yr}^{-1} \mathrm{kpc}^{-2}}\right)^{-0.14} R_{6} v_{250}^{-1} .
$$

Since the above estimate comes mainly from dynamical measurements and gas fractions calibrated on $\mathrm{CO}$ observations from gasrich, $z \sim 0-3$ mergers ( Tacconi et al. 2008), the above expression would appear to be relatively immune to changes in the assumed initial mass function. With the Bouché et al. (2007) calibration of the Kennicutt-Schmidt relation, the gas fractions in the five galaxies we have studied range between 0.1 and 0.3 of the dynamical masses (Table 2), and the gas exhaustion timescales, $M_{\text {gas }} / \mathrm{SFR}(\mathrm{H} \alpha)$, are $\sim 2 \times 10^{8} \mathrm{yr}$, significantly smaller than the SED ages or the stellar buildup ages, $M_{*} / \operatorname{SFR}(\mathrm{H} \alpha)$. As it is highly unlikely that all BX/BzK galaxies are about to run out of gas and will soon stop forming stars, this discrepancy argues for a continuous gas supply (Erb 2008), and thus is consistent with the continuous gas accretion model. However, the gas masses in Table 2 are obviously based on a somewhat uncertain, indirect method. Direct measurements of gas masses from $\mathrm{CO}$ rotational line luminosities would be highly desirable. First CO observations in a few $\mathrm{BX} / \mathrm{BzK}$ galaxies have resulted in detections in two cases (possibly implying much larger gas masses than in Table 2; Daddi et al. 2008) and upper limits in three other cases (Tacconi et al. 2008). These initial observations do not yet provide a clear-cut test of whether the Bouché et al. (2007) calibration is appropriate, especially when taking into account the uncertainty in the $\mathrm{CO}$-luminosity-to- $\mathrm{H}_{2}$-gas mass conversion factor (see Tacconi et al. 2008 for a discussion).

The combination of efficient secular evolution and higher efficiency star formation may thus be able to account for the observed rapid buildup of stellar mass at high redshift. It needs to be explored whether secular evolution may also be able to influence the buildup of the central massive black holes and to account for the black hole mass to bulge mass (or velocity dispersion) relation.

\section{CONCLUSIONS}

We have presented high-quality SINFONI/VLT integral field spectroscopy of five large $M \sim 10^{11} M_{\odot}$ disk galaxies at $z \sim 2$. Aided in part by laser guide star adaptive optics, our data constrain the galaxy dynamics on scales of $\leqslant 3 \mathrm{kpc}$ and allow us to detect and estimate the dynamical mass of the central bulge/inner disk, in addition to that of the outer disk.

We find evidence for a substantial central bulge and/or central disk in four of the five cases, whose mass fraction relative to that

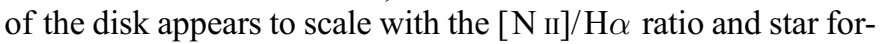
mation age of the galaxies.

Interpreting these observations in terms of an evolutionary sequence, we propose that massive bulges/central disks at $z \sim$ $2-3$ can grow on a timescale of $\leqslant 1$ Gyr through secular evolution of early turbulent, gas-rich disks. Numerical simulations and simple analytical estimates suggest that clumpy, turbulent disks may be a transient phase in the early evolution of most, if not all, galaxies that are going through a phase of rapid "cold" accretion from the surrounding halos. To a significant extent, the large turbulence may be stirred up by the kinetic energy of the accreting gas itself. Precisely because of the large turbulence, internal secular disk evolution then proceeds on a timescale $\leqslant 1$ Gyr, an order of magnitude more rapidly than at $z \sim 0$. After the clumpy, turbulent phase is ended, the product may be 
a (massive) central bulge surrounded by a remnant thick exponential disk whose $z \sim 0$ relic is the old thick disk component seen in many nearby galaxies. As a result of the cosmological evolution of accretion rates combined with the ceasing of rapid cold flows once halos grow above $\sim 10^{11.5}-10^{12} M_{\odot}$, accretion rates strongly decrease with decreasing redshift, such that the disk turbulence subsides and maturing thin disks grow at $z \leqslant 1$.

These secular effects, along with more efficient star formation at high gas surface densities, may also help to account for the $\sim 1$ Gyr timescales for the buildup of the stellar component that appears to be typical at $z \sim 2$. One is left to wonder whether the same secular processes also help build up central black holes in this epoch of maximum QSO activity.

We thank the staff of Paranal Observatory for their support. This work would have been impossible without the dedicated work of the ESO LGSF team at ESO, MPE and MPIA Heidelberg. We are grateful to Norm Murray, Eve Ostriker, and Chris McKee for enlightening discussions on star formation processes and to Avishai Dekel and an anonymous referee for valuable comments on the paper.
Abadi, M. G., Navarro, J. F., Steinmetz, M., \& Eke, V. R. 2003, ApJ, 591, 499 Adelberger, K. L., Steidel, C. C., Shapley, A. E., Hunt, M. P., Erb, D. K., Reddy, N. A., \& Pettini, M. 2004, ApJ, 607, 226

Bett, P., Eke, V., Frenk, C. S., Jenkins, A., Helly, J., \& Navarro, J. 2007, MNRAS, 376, 215

Binney, J., \& Tremaine, S. 2008, Galactic Dynamics (2nd ed.; Princeton: Princeton Univ. Press)

Birnboim, Y., \& Dekel, A. 2003, MNRAS, 345, 349

Birnboim, Y., Dekel, A., \& Neistein, E. 2007, MNRAS, 380, 339

Bonaccini Calia, D., et al. 2006, Proc. SPIE, 6272, 6

Bonnet, H., et al. 2004, ESO Messenger, 117, 17

Bouché, N., et al. 2007, ApJ, 671, 303

Bournaud, F., \& Combes, F. 2002, A\&A, 392, 83

Bournaud, F., Elmegreen, B. G., \& Elmegreen, D. M. 2007, ApJ, 670, 237

Bower, R. G., et al. 2006, MNRAS, 370, 645

Bullock, J. S., Kolatt, T. S., Sigad, Y., Somerville, R. S., Kravtsov, A. V., Klypin, A. A., Primack, J. R., \& Dekel, A. 2001, MNRAS, 321, 559

Calzetti, D. 2001, PASP, 113, 1449

Chabrier, G. 2003, PASP, 115, 763

Conroy, C., Shapley, A. E., Tinker, J. L., Santos, M. R., \& Lemson, G. 2008, ApJ, 679, 1192

Cowie, L., Hu. E., \& Songaila, A. 1995, AJ, 110, 1576

Daddi, E., Cimatti, A., Renzini, A., Fontana, A., Mignoli, M., Pozetti, L., Tozzi, P., \& Zamorani, G. 2004a, ApJ, 617, 746

Daddi, E., Dannerbauer, H., Elbaz, D., Dickinson, M., Morrison, G., Stern, D., \& Ravindranath, S. 2008, ApJ, 673, L21

Daddi, E., et al. 2004b, ApJ, 600, L127 2007, ApJ, 670, 156

Davé, R. 2008, MNRAS, 385, 147

Dekel, A., \& Birnboim, Y. 2006, MNRAS, 368, 2

Dekel, A., \& Silk, J. 1986, ApJ, 303, 39

Dekel, A., \& Woo, J. 2003, MNRAS, 344, 1131

Dib, S., Bell, E., \& Burkert, A. 2006, ApJ, 638, 797

Efstathiou, G. 2000, MNRAS, 317, 697

Eisenhauer, F., et al. 2003, Proc. SPIE, 4841, 1548

Elbaz, D., et al. 2007, A\&A, 468, 33

Elmegreen, B. G., \& Elmegreen, D. M. 2005, ApJ, 627, 632

. 2006, ApJ, 650, 644

Elmegreen, D. M., Elmegreen, B. G., Ravindranath, S., \& Cox, D. A. 2007, ApJ, 658, 763

Erb, D. K. 2008, ApJ, 674, 151

Erb, D. K., Shapley, A. E., Pettini, M., Steidel, C. C., Reddy, N. A.\& Adelberger, K. L. 2006a, ApJ, 644, 813

Erb, D. K., Steidel, C. C., Shapley, A. E., Pettini, M., Reddy, N. A., \& Adelberger, K. L. 2006b, ApJ, 646, 107 2006c, ApJ, 647, 128

Fakhouri, O., \& Ma, C. P. 2008, MNRAS, 386, 577

Förster Schreiber, N. M., et al. 2004, ApJ, 616, 40 2006, ApJ, 645, 1062 2008, ApJ, submitted

Franx, M., et al. 2003, ApJ, 587, L79

Freeman, K., \& Bland-Hawthorn, J. 2002, ARA\&A, 40, 487

Genel, S., et al. 2008, ApJ, in press (arXiv:0808.0194)

Genzel, R., et al. 2006, Nature, 442, 786

Grazian, A., et al. 2007, A\&A, 465, 393

Guo, Q., \& White, S. D. M. 2008, MNRAS, 384, 2
Immeli, A., Samland, M. Gerhard, O., \& Westera, P. 2004a, A\&A, 413, 547 Immeli, A., Samland, M., Westera, P. \& Gerhard, O. 2004b, ApJ, 611, 20

Kennicutt, R. C. Jr. 1998, ApJ, 498, 541

Keres, D., Katz, N., Weinberg, D. H., \& Davé, R. 2005, MNRAS, 363, 2

Kitzbichler, M. G., \& White, S. D. M. 2007, MNRAS, 376, 2

Kong, X., et al. 2006, ApJ, 638, 72

Kormendy, J., \& Kennicutt, R. C., Jr. 2004, ARA\&A, 42, 603

Labbé, I., et al. 2003, ApJ, 591, L95

Law, D., Steidel, C. C., Erb, D. K., Larkin, J. E., Pettini, M., Shapley, A. E., \& Wright, S. A. 2007, ApJ, 669, 929

Lin, D. N. C., \& Pringle, J. E. 1987, ApJ, 320, L87

Melendez, J., et al. 2008, A\&A, 484, L21

Mo, H. J., Mao, S., \& White, S. D. M. 1998, MNRAS, 295, 319

Monaco, P. 2004, MNRAS, 352, 181

Naab, T., Johansson, P. H., Ostriker, J. P., \& Efstathiou, G. 2007, ApJ, 658, 710

Navarro, J. F., Frenk, C. S., \& White, S. D. M. 1997, ApJ, 490, 493

Noeske, K. G., et al. 2007, ApJ, 660, L43

Noguchi, M. 1999, ApJ, 514, 77

Ocvirk, P., Pichon, C., \& Tessier, R. 2008, MNRAS, in press (arXiv:0803.4506)

Pettini, M., \& Pagel, B. E. J. 2004, MNRAS, 348, L59

Rabien, S., et al. 2003, Proc. SPIE, 4839, 393

Reddy, N. A., Erb, D. K.,Steidel, C. C., Shapley, A. E., Adelberger, K. L., \& Pettini, M. 2005, ApJ, 633, 748

Rudnick, G., et al. 2006, ApJ, 650, 624

Samland, M., \& Gerhard, O. 2003, A\&A, 399, 961

Schreiber, J., Thatte, N., Eisenhauer, F., Tecza, M., Abuter, R., \& Horrobin, M. 2004, ASP Conf. Ser. 314, Astronomical Data Analysis Software and Systems XIII (San Francisco: ASP), 380

Semelin, B., \& Combes, F. 2002, A\&A, 388, 826

Shakura, N. I., \& Sunyaev, R. A. 1973, A\&A, 24, 337

Shapiro, K., et al. 2008, ApJ, 682, 231

Shapley, A. E., et al. 2005, ApJ, 626, 698

Silk, J. 2001, MNRAS, 324, 313

Silk, J., \& Norman, C. 1981, ApJ, 247, 59

Springel, V., et al. 2005, Nature, 435, 629

Steidel, C. C., Giavalisco, M., Pettini, M., Dickinson, M., \& Adelberger, K. L. 1996, ApJ, 462, L17

Steidel, C. C., Shapley, A. E., Pettini, M., Adelberger, K. L., Erb, D. K., Reddy, N. A., \& Hunt, M. P. 2004, ApJ, 604, 534

Steinmetz, M., \& Navarro, J. F. 2002, NewA, 7, 155

Stockton, A., McGrath, E., Canalizo, G. Iye, M., \& Maihara, T. 2008, ApJ, 672, 146

Swaters, R. A., Schoenmakers, R. H. M., Sancisi, R., \& van Albada, J. S. 1999, MNRAS, 304, 330

Swinbank, A. M., et al. 2006, MNRAS, 371, 465

Tacconi, L. J., Neri, R., Chapman, S. C., Genzel, R., Smail, I., Ivison, R. J., Bertoldi, F., Blain, A., Cox, P., Greve, T., \& Omont, A. 2006, ApJ, 640, 228 Tacconi, L. J., et al. 2008, ApJ, 680, 246

Thompson, T., Quataert, E., \& Murray, N. 2005, ApJ, 630, 167

van den Bergh, S., Abraham, R. G., Ellis, R. S., Tanvir, N. R., Santiago, B. X., \& Glazebrook, K. G. 1996, AJ, 112, 359

van der Kruit, P. C., \& Allen, R. J. 1978, ARA\&A, 16, 103

Wright, S. A., et al. 2007, ApJ, 658, 78

Yoachim, P., \& Dalcanton, J. J. 2006, AJ, 131, 226

Zoccali, M., et al. 2003, A\&A, 399, 931 